\title{
Standard Molar Enthalpies of Formation of Halomethanes Based on Quantum Chemical Computations
}

\author{
K. G. Kalamatianos \\ Physical and Computational Chemistry Laboratory, I.Y.A., Athens, Greece
}

\section{ABSTRACT}

Accurate calculations of standard molar enthalpies of formation $\left(\Delta \mathrm{H}_{\mathrm{f}}{ }^{\circ}\right)_{\mathrm{m}}(\mathrm{g})$ and carbonhalogen bond dissociation enthalpies, $\mathrm{BDE}$, of a variety of halomethanes with relevance on several atmospheric chemical processes and particularly to ozone destruction, were performed in the gas phase at $298.15 \mathrm{~K}$. The $\left(\Delta \mathrm{H}_{\mathrm{f}}{ }^{\circ}\right)_{\mathrm{m}}(\mathrm{g})$ of the radicals formed through bond dissociations have also been computed. $A b$ initio computational methods and isodesmic reaction schemes were used. It is found that for the large majority of these species, the gold standard method of quantum chemistry $(\operatorname{CCSD}(T))$ and even MP2 are capable to predict enthalpy values nearing chemical accuracy provided that isodesmic reaction schemes are used. New estimates for standard molar enthalpies of formation and $\mathrm{BDE}$ are suggested including for species that to our knowledge there are no experimental $\left(\Delta \mathrm{H}_{\mathrm{f}}{ }^{\circ}\right)_{\mathrm{m}}(\mathrm{g})\left(\mathrm{CHCl}_{2} \mathrm{Br}, \mathrm{CHBr}_{2} \mathrm{Cl}, \mathrm{CHBrCl}, \mathrm{CHICl}, \mathrm{CHIBr}\right)$ or $\mathrm{BDE}$ values $\left(\mathrm{CHCl}_{2} \mathrm{Br}, \mathrm{CHBr}_{2} \mathrm{Cl}, \mathrm{CHBrCl}, \mathrm{CHICl}, \mathrm{CHIBr}\right)$ available in the literature. The method and calculational procedures presented may profitably be used to obtain accurate $\left(\Delta \mathrm{H}_{\mathrm{f}}{ }^{\circ}\right)_{\mathrm{m}}(\mathrm{g})$ and BDE values for these species.

\section{INTRODUCTION}

A plethora of factors has generated considerable interest in recent years in establishing highly accurate standard molar enthalpies of formation $\left(\Delta \mathrm{H}_{\mathrm{f}}{ }^{\circ}\right)_{\mathrm{m}}(\mathrm{g})$, bond energies and heat capacities. Among these are the following: i) the advances in quantum mechanical calculations which are now capable of achieving $\pm 1 \mathrm{~kJ} \mathrm{~mol}^{-1}$ accuracy for small molecules ${ }^{1-4}$ ii) prediction of thermochemistry is crucial for designing chemicals with new functionality since fundamental properties of compounds such as enthalpy, heat capacity, standard entropy and Gibbs free energy are needed to understand stability and/or reactivity ${ }^{5-8}$ iii) accurate, reliable and internally consistent thermochemistry is a necessary condition in many fields of physical chemistry, ranging from kinetics and reaction mechanisms to flames and atmospheric chemistry ${ }^{9}$ iv) the emergence of the active thermochemical tables - that are based on experimental data and theoretical calculations - a novel approach of how to obtain accurate, reliable, and internally consistent thermochemical values and the fact that are rapidly becoming the archetypal approach to thermochemistry ${ }^{9} \quad$ v) several databases containing relevant thermochemical data for halomethanes $-\mathrm{NIST}^{10}$, Pedley ${ }^{11}, \mathrm{JPL}^{12}$, Luo ${ }^{13}$, Kurdchaker and Kurdchaker ${ }^{14}$, Gurvich et al. ${ }^{15}$ - have considerably large error bars or are based on inaccurate experimental information vi) the scarcity of experimental data that is 
particularly severe for bromine and iodine containing halomethane species vii) halomethanes such as $\mathrm{CH}_{2} \mathrm{Br}_{2}, \mathrm{CH}_{2} \mathrm{I}_{2}, \mathrm{CH}_{2} \mathrm{BrI}, \mathrm{CH}_{2} \mathrm{ICl}, \mathrm{CHClBr}_{2}$ and $\mathrm{CHCl}_{2} \mathrm{Br}$ and others have been observed in the troposphere, and consequently have been considered important sources of reactive halogens in the atmosphere. ${ }^{16-19}$ In this context, quantum mechanical calculations of thermochemical properties of halogenated organic compounds is of importance. Models using methodologies such as isodesmic reaction schemes and/or atomization and quantum chemical calculations are widely applied in predicting thermochemical values. The ab initio Gaussian-n $(G n)^{20-22}$, Weizman- $n^{23}$, and Petersson-style $^{24}$ complete basis set models have improved accuracy in ab initio thermochemistry by combining calculations at different levels of theory and basis sets with empirical corrections in most methods. The empirical corrections limit their predictive capability to the datasets against which they are benchmarked ${ }^{25}$. Furthemore, Gn methods are available for chlorine and bromine, but not for iodine.

The present work has the following objectives:

i) to reproduce the best experimental available values for the standard molar enthalpies of formation $\left(\Delta \mathrm{H}_{\mathrm{f}}{ }^{\circ}\right)_{\mathrm{m}}(\mathrm{g})$ of a set of halomethanes by means of isodesmic reactions using $a b$ initio calculations and the DFT, MP2 and $\operatorname{CCSD}(\mathrm{T})$ methods

ii) to obtain the corresponding correlation equations by plotting experimental versus theoretical values of $\left(\Delta \mathrm{H}_{\mathrm{f}}{ }^{\circ}\right)_{\mathrm{m}}(\mathrm{g})$ for the halomethanes in (i)

iii) to evaluate the standard molar enthalpy of formation $\left(\Delta \mathrm{H}_{\mathrm{f}}{ }^{\circ}\right)_{\mathrm{m}}(\mathrm{g})$ of halomethanes where experimental data are either not available - iodine containing or mixed halomethanes - or inconsistent and/or error bars are large ( $>10 \mathrm{~kJ} \mathrm{~mol}^{-1}$ ) using the theoretical calculations in (i) and the corresponding correlation equations in (ii)

iv) To determine the accuracy of the proposed method for predicting standard molar enthalpies of formation $\left(\Delta \mathrm{H}_{\mathrm{f}}{ }^{\circ}\right)_{\mathrm{m}}(\mathrm{g})$ of halomethanes

v) to provide existing databases of thermochemical properties with new and more reliable standard molar enthalpies of formation of these species

The set of the studied halomethanes are shown in Table $1 A \& 1 B$. Several of these species and their breakdown products contribute to ozone destruction in both the troposphere and stratosphere ${ }^{26}$. Mixed trihalomethanes $\left(\mathrm{CHBrCl}_{2}, \mathrm{CHClBr}_{2}\right)$ are also major organic by-products of drinking water chlorination, resulting from the reaction of chlorine with natural organic material and bromine in source waters. The discovery of these by-products in drinking water has raised questions about their toxicity ${ }^{27}$.

\section{COMPUTATIONAL METHODS}

The ab initio molecular orbital calculations were carried out using the Firefly Quantum 
Chemistry Package version 8.2.0 28 , 29 the GAMESS US program ${ }^{29,30}$ and the ORCA 4.1 quantum chemistry program package ${ }^{31}$. Optimization and frequency calculations of the compounds studied have been carried out with several methods, such as density functional theory (DFT) with the B3LYP ${ }^{31}$ functional and the ADZP basis set ${ }^{32-34}, M P 2$, $\operatorname{CCSD}(T)^{35}$ and DLPNO-CCSD $(T)^{36-37}$ and the SPK-ATZP basis set. ${ }^{38-39}$ Zero-point $^{35}$ energies and corrections to enthalpies at 298.15 were added from geometries and frequencies at the level of the geometry optimization. Isodesmic reactions (R1-R3) were used for the calculation of standard molar enthalpies of formation $\left(\Delta \mathrm{H}_{\mathrm{f}}{ }^{\circ}\right)_{\mathrm{m}}(\mathrm{g})$ since these has been shown to be more accurate than atomization reactions ${ }^{40-42}$ due to the cancellation of errors involving similar chemical bonds:

$$
\begin{aligned}
& \mathrm{CHX}_{2} \mathrm{Z}+2 \mathrm{CH}_{4} \rightarrow \mathrm{CH}_{3} \mathrm{Z}+2 \mathrm{CH}_{3} \mathrm{X} \text { (where } \mathrm{X}, \mathrm{Z}=\mathrm{F}, \mathrm{Cl}, \mathrm{Br}, \mathrm{I} \text { ) } \\
& \mathrm{CHX}_{2} \mathrm{Z}+\mathrm{CH}_{4} \rightarrow \mathrm{CH}_{3} \mathrm{Z}+\mathrm{CH}_{2} \mathrm{X}_{2} \text { (where } \mathrm{X}, \mathrm{Z}=\mathrm{F}, \mathrm{Cl}, \mathrm{Br}, \mathrm{I} \text { ) } \\
& \mathrm{CHX}_{2} \mathrm{Z}+\mathrm{CH}_{4} \rightarrow \mathrm{CH}_{2} \mathrm{XZ}+\mathrm{CH}_{3} \mathrm{X} \text { (where } \mathrm{X}, \mathrm{Z}=\mathrm{F}, \mathrm{Cl}, \mathrm{Br}, \mathrm{I} \text { ) }
\end{aligned}
$$

Throughout this paper, standard molar enthalpies of formation and reaction are given in $\mathrm{kJ} . \mathrm{mol}^{-1}$ at a temperature of $298.15 \mathrm{~K}$.

\section{RESULTS AND DISCUSSION}

The available experimental values of standard molar enthalpies of formation $\left(\Delta \mathrm{H}_{\mathrm{f}}{ }^{\circ}\right)_{\mathrm{m}}(\mathrm{g})$ for the set of halomethanes studied in this work are summarized in Table $1 \mathrm{~A} \& 1 \mathrm{~B}$. Values of $\left(\Delta \mathrm{H}_{\mathrm{f}}{ }^{\circ}\right)_{\mathrm{m}}(\mathrm{g})$ collected in reviews and evaluations and from the Argonne Thermochemical Network ${ }^{43}$ are also included.

As shown, there is poor agreement between experimental $\left(\Delta \mathrm{H}_{\mathrm{f}}{ }^{\circ}\right)_{\mathrm{m}}(\mathrm{g})$ values in several species. The following experimental values are reported for $\mathrm{CHBr}_{3}$ (Table $1 \mathrm{~A} \&$ \& $\mathrm{B}$ ): 55.1 $\pm 4.9^{44}, 55.4 \pm 3.3^{45}, 23.8 \pm 4.5^{11} \mathrm{~kJ} \mathrm{~mol}^{-1}$. Similarly, the experimental enthalpy of formation values of $\mathrm{CH}_{2} \mathrm{BrCl}$ differ considerably $-44.1 \pm 1.9^{46}$ and $-20 \pm 7^{10},-45.0 \pm 5^{15}$.

The $\left(\Delta \mathrm{H}_{\mathrm{f}}{ }^{\circ}\right)_{\mathrm{m}}(\mathrm{g})$ values reported for iodine-containing halomethanes (Table $1 \mathrm{~A}$ ) have large error margins. The value reported for $\mathrm{CHCl}_{2} \mathrm{I}$ by Gurvich et al. ${ }^{15}$ is $5.0 \pm 20 \mathrm{~kJ} . \mathrm{mol}^{-1}$ while for $\mathrm{CHBr}_{2} \mathrm{I}$ is $110.0 \pm 25 \mathrm{~kJ} \cdot \mathrm{mol}^{-1}$.

The standard molar enthalpies of formation of mixed trihalomethanes are much less well established. Experimental ${ }^{44}$ and computed values ${ }^{47}$ of only two species $\mathrm{CHBr}_{2} \mathrm{Cl}$ and $\mathrm{CHCl}_{2} \mathrm{Br}$ have been recently determined which is surprising considering the importance of these molecules in atmospheric chemistry.

Computed values $\left(\Delta \mathrm{H}_{\mathrm{f}}{ }^{\circ}\right)_{\mathrm{m}}(\mathrm{g})$ of the studied compounds using the methods described above and isodesmic reactions (R1-R3) are collected in Table $1 \mathrm{~A}$ and $1 \mathrm{~B}$. As shown, the mean absolute error (MAE) between experimental and computed values of the species 
TABLE 1A: $\left(\Delta \mathrm{Hf}^{\circ}\right)_{\mathrm{m}}(\mathrm{g})$ values of halomethanes, experimental and computed at DFT/SPK-ATZP and MP2/SPK-ATZP levels of theory.

\section{$\left(\Delta \mathrm{H}_{\mathrm{f}}^{\circ}\right)_{\mathrm{m}}(\mathrm{g}) \mathrm{kJ}_{\mathrm{mol}} \mathrm{mo}^{-1}(298.15 \mathrm{~K})$}

\begin{tabular}{|c|c|c|c|c|c|c|c|c|c|c|}
\hline & \multirow[t]{2}{*}{ Species } & \multicolumn{3}{|c|}{ DFT/SPK-ATZP } & \multicolumn{3}{|c|}{ MP2/SPK-ATZP } & \multirow[b]{2}{*}{$\begin{array}{l}\text { Experim. } \\
(E X)^{b}\end{array}$} & \multirow[b]{2}{*}{$\begin{array}{l}\text { Eval. } \\
(E V)^{b}\end{array}$} & \multirow[b]{2}{*}{ EX-EV } \\
\hline & & $\mathbf{R 1}^{a, c}$ & $\mathbf{R} \mathbf{2}^{a, c}$ & $\mathbf{R 3}^{a, c}$ & $\mathbf{R 1}^{a, c}$ & $\mathbf{R 2}^{a, c}$ & $\mathbf{R 3}^{a, c}$ & & & \\
\hline 1 & $\mathrm{CHBr}_{3}$ & $61.4(-13.3)$ & $59.0(-10.9)$ & & $43.6(4.5)$ & $46.4(1.7)$ & & $\begin{array}{c}\mathbf{4 8 . 1 0}[\mathbf{1 . 3}]^{f} \\
55.1\left[^{\prime} \cdot 9\right]^{d}, 55.4 \\
{[3.3]^{e}, 23.8[4.5]^{g}}\end{array}$ & $47.4 \pm 2.6$ & 0.7 \\
\hline 2 & $\mathrm{CH}_{3} \mathrm{Br}$ & $-39.8(2.1)$ & $-41.6(3.9)$ & & $-33.9(-3.8)$ & $-29.0(-8.7)$ & & 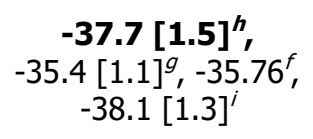 & $-35.5 \pm 6.3$ & -2.2 \\
\hline 3 & $\mathrm{CH}_{2} \mathrm{Br}_{2}$ & $-0.8(4)$ & $-0.7(3.9)$ & & $0.4(2.8)$ & $11.9(-8.7)$ & & $\begin{array}{c}\mathbf{3 . 2}[\mathbf{3 . 4}]^{k} \\
3.5^{f}, 10.0[15]^{\prime}\end{array}$ & $3.6 \pm 6.5$ & -0.4 \\
\hline 4 & $\mathrm{CHCl}_{3}$ & $-87.2(-15.7)$ & $-93.5(-9.4)$ & & $-99.6(-3.3)$ & $-102.4(-0.5)$ & & $\begin{array}{l}\mathbf{- 1 0 2 . 9}[\mathbf{2 . 5}]^{\mathbf{m}} \\
-103.2^{n},-103.39^{f}\end{array}$ & $-98.0 \pm 4.6$ & -4.9 \\
\hline 5 & $\mathrm{CH}_{3} \mathrm{Cl}$ & $-87.1(5.2)$ & $-91.3(9.4)$ & & $-83.0(1.1)$ & $-82.4(0.5)$ & & $\begin{array}{c}\mathbf{- 8 1 . 9}[\mathbf{1 . 5}]^{\mathbf{m}} \\
-81.9[0.5]^{k},- \\
82.18^{f}\end{array}$ & $-82.9 \pm 0.4$ & 1.0 \\
\hline 6 & $\mathrm{CH}_{2} \mathrm{Cl}_{2}$ & & $-105.1(9.6)$ & & & $-96.2(0.5)$ & & 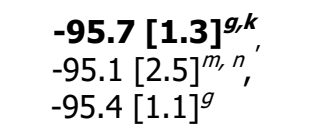 & $-96.6 \pm 0.5$ & 0.9 \\
\hline 7 & $\mathrm{CH}_{2} \mathrm{BrCl}$ & $-42.0(-2.1)$ & $-45.1(1.0)$ & & $-45.8(1.7)$ & $-47.2(3.1)$ & $-45.8(1.7)$ & $\begin{array}{c}\mathbf{- 4 4 . 1}\left[\mathbf{1 . 9 ] ^ { k }}{ }^{\prime}\right. \\
-20[7]^{m},-43.3^{f} \\
-45.0[5]^{\prime}\end{array}$ & $-45.9 \pm 0.9$ & 1.8 \\
\hline
\end{tabular}


$\left(\Delta \mathrm{H}_{\mathrm{f}}{ }^{\circ}\right)_{\mathrm{m}}(\mathrm{g}) \mathrm{kJ} \cdot \mathrm{mol}^{-1}(298.15 \mathrm{~K})$

\begin{tabular}{|c|c|c|c|c|c|c|c|c|c|c|}
\hline & \multirow[t]{2}{*}{ Species } & \multicolumn{3}{|c|}{ DFT/SPK-ATZP } & \multicolumn{3}{|c|}{ MP2/SPK-ATZP } & \multirow[b]{2}{*}{$\begin{array}{c}\text { Experim. } \\
(E X)^{b}\end{array}$} & \multirow[b]{2}{*}{$\begin{array}{l}\text { Eval. } \\
(\mathrm{EV})^{b}\end{array}$} & \multirow[b]{2}{*}{ EX-EV } \\
\hline & & $\mathbf{R 1}^{a, c}$ & $\mathbf{R} 2^{a, c}$ & $\mathbf{R 3}^{a, c}$ & $\mathbf{R 1}^{a, c}$ & $\mathbf{R} 2^{a, c}$ & $\mathbf{R 3}^{a, c}$ & & & \\
\hline 8 & $\mathrm{CHCl}_{2} \mathrm{~F}$ & $-273.5(-9.8)$ & $-279.8(-3.5)$ & $-279.8(-3.5)$ & $-279.6(-3.7)$ & $-282.4(-0.9)$ & $-282.4(-0.9)$ & $\begin{array}{l}-283.3[13]^{p, q} \\
-284.20^{f},-284.1^{\prime}\end{array}$ & $-282.8 \pm 1.2$ & -0.5 \\
\hline 9 & $\mathrm{CHCl}_{2} \mathrm{Br}$ & $-37.9(-12.2)$ & $-44.2(-5.9)$ & $-44.2(-5.9)$ & $-51.1(1.0)$ & $-53.8(3.7)$ & $-49.3(-0.8)$ & $\begin{array}{c}-50.1[\mathbf{1 . 8}]^{d}{ }^{\prime} \\
-50.3^{f},-50.9[2.4]^{x o}\end{array}$ & $-48.2 \pm 3.2$ & -1.9 \\
\hline 10 & $\mathrm{CHBr}_{2} \mathrm{Cl}$ & $11.6(-11.6)$ & $9.3(-9.3)$ & $9.5(-9.5)$ & $-3.3(3.3)$ & $-0.5(0.5)$ & $-1.6(1.6)$ & $\begin{array}{l}\mathbf{0 . 0}\left[\mathbf{3 . 4}^{f f}\right. \\
4.1^{d}, 10.0^{\prime}\end{array}$ & $-0.8 \pm 1.0$ & 0.8 \\
\hline 11 & $\mathrm{CFCl}_{3}$ & & & & $-283.7(-5.0)$ & $-287.9(-0.8)$ & $-287.4(-1.3)$ & $\begin{array}{l}\mathbf{- 2 8 8 . 7}[\mathbf{6 . 3}]^{p} \\
-290.1^{f},-278^{s^{\prime}}\end{array}$ & $-287.7 \pm 1.3$ & -1 \\
\hline 12 & $\mathrm{CF}_{3} \mathrm{Br}$ & & & & & $-647.9(-1.0)$ & $-651.6(2.7)$ & $\begin{array}{l}\mathbf{- 6 4 8 . 9}[\mathbf{2 . 9}]^{p} \\
-647.0^{t},-651.57^{f}\end{array}$ & $-648.3 \pm 5.0$ & -0.6 \\
\hline 13 & $\mathrm{CF}_{2} \mathrm{Cl}_{2}$ & & & & $-485.5(-6.1)$ & $-488.2(-3.4)$ & $-489.1(-2.5)$ & $\begin{array}{c}-491.6[8]^{q} \\
-495.46^{f},-477^{\prime} .6^{i}\end{array}$ & $-491.5 \pm 1.2$ & -0.1 \\
\hline 14 & $\mathrm{CHF}_{2} \mathrm{Cl}$ & $-469.2(-12.4)$ & $-478.0(-3.6)$ & $-470.7(-10.9)$ & $-475.4(-6.2)$ & $-480.1(-1.5)$ & $-476.4(-5.2)$ & 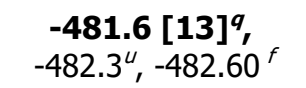 & $-480.1 \pm 3.4$ & -1.5 \\
\hline 15 & $\mathrm{CH}_{2} \mathrm{ClF}$ & $-260.4(-1.5)$ & $-263.6(1.7)$ & & $-261.0(-0.9)$ & $-262.4(0.5)$ & & $\begin{array}{c}-\mathbf{2 6 1 . 9}[13]^{q} \\
-263.60^{f}\end{array}$ & $-264.3 \pm 1.9$ & 2.4 \\
\hline 16 & $\mathrm{CF}_{3} \mathrm{Cl}$ & & & & $-697.6(-12.4)$ & $-704.7(-5.3)$ & $-708.3(-1.7)$ & $\begin{array}{c}\mathbf{- 7 1 0 . 0}[\mathbf{3 . 3}]^{q} \\
-710.08^{f},-707.3^{w}\end{array}$ & $-708.9 \pm 3.5$ & -1.1 \\
\hline
\end{tabular}


$\left(\Delta \mathrm{H}_{\mathrm{f}}{ }^{\circ}\right)_{\mathrm{m}}(\mathrm{g}) \mathrm{kJ} \cdot \mathrm{mol}^{-1}(298.15 \mathrm{~K})$

\begin{tabular}{|c|c|c|c|c|c|c|c|c|c|c|}
\hline & \multirow[t]{2}{*}{ Species } & \multicolumn{3}{|c|}{ DFT/SPK-ATZP } & \multicolumn{3}{|c|}{ MP2/SPK-ATZP } & \multirow[b]{2}{*}{$\begin{array}{c}\text { Experim. } \\
(E X)^{b}\end{array}$} & \multirow[b]{2}{*}{$\begin{array}{l}\text { Eval. } \\
\text { (EV) }^{b}\end{array}$} & \multirow[b]{2}{*}{ EX-EV } \\
\hline & & $\mathbf{R 1}^{a, c}$ & $\mathbf{R} \mathbf{2}^{a, c}$ & $\mathbf{R 3}^{a, c}$ & $\mathbf{R 1}^{a, c}$ & $\mathbf{R} \mathbf{2}^{a, c}$ & $\mathbf{R 3}^{a, c}$ & & & \\
\hline 17 & $\mathrm{CH}_{2} \mathrm{ICl}$ & $8.0(2.7)$ & $8.9(1.8)$ & $8.0(2.7)$ & $11.3(-0.6)$ & $9.9(0.8)$ & & $10.7[1.9]^{k}, 13.6^{v}$ & $9.6 \pm 2.9$ & 1.1 \\
\hline 18 & $\mathrm{CH}_{2} \mathrm{IBr}$ & $59.0(-4.0)$ & $59.8(-4.8)$ & & $56.0(-1.0)$ & $55.7(-0.7)$ & & $55.0[3.4]^{k}, 57.1^{v}$ & $58.0 \pm 2.3$ & -3.0 \\
\hline 19 & $\mathrm{CHI}_{2} \mathrm{Cl}$ & $112.9(-2.9)$ & $114.6(-4.6)$ & & $110.6(-0.6)$ & $109.2(0.8)$ & $110.0(0)$ & $\mathbf{1 1 0 . 0}[\mathbf{3 0}]^{\prime}, 112.9^{v}$ & $113.5 \pm 2.3$ & -3.5 \\
\hline 20 & $\mathrm{CHI}_{2} \mathrm{Br}$ & $168.9(-3.9)$ & $167.7(-2.7)$ & & $155.1(9.9)$ & $163.5(1.5)$ & & $\begin{array}{c}\mathbf{1 6 5 . 0}[\mathbf{3 5}]^{\prime}, \\
158.20^{v}\end{array}$ & $165.4 \pm 4.7$ & -0.4 \\
\hline 21 & $\mathrm{CHCl}_{2} \mathrm{I}$ & $7.2(-2.2)$ & $0.9(4.1)$ & $9.9(-4.9)$ & $7.7(-2.7)$ & $4.9(0.1)$ & $7.1(-2.1)$ & $5.0[20]^{\prime}, 8.28^{v}$ & $7.0 \pm 2.3$ & -2.0 \\
\hline 22 & $\mathrm{CHBr}_{2} \mathrm{I}$ & $113.2(-3.2)$ & $110.9(-0.9)$ & $109.3(0.7)$ & $100.0(10.0)$ & $104.2(5.8)$ & $99.0(11.0)$ & $\mathbf{1 1 0 . 0}[\mathbf{2 5}]^{\prime}, 93.51^{v}$ & $105.6 \pm 4.0$ & 4.4 \\
\hline 23 & $\mathrm{CF}_{2} \mathrm{ClI}$ & $-376.6(-3.4)$ & $-382.7(2.7)$ & $-389.0(9)$ & $-366.0(-14.0)$ & $-371.3(-8.7)$ & $-372.2(-7.8)$ & $\begin{array}{c}\mathbf{- 3 8 0 . 0 [ 2 5}]^{\prime} \\
.379 .07^{l}\end{array}$ & $-372.3 \pm 1.3$ & -7.7 \\
\hline 24 & $\mathrm{CF}_{2} \mathrm{BrI}$ & $-320.9(-4.1)$ & $-327.0(2)$ & $-327.8(2.8)$ & $-316.1(-8.9)$ & $-317.8(-7.2)$ & $-318.4(-6.6)$ & $\begin{array}{c}\mathbf{- 3 2 5 . 0}[\mathbf{2 5}]^{\prime} \\
-324.30^{v}\end{array}$ & $-325.1 \pm 6.0$ & 0.1 \\
\hline 25 & $\mathrm{CFCl}_{2} \mathrm{I}$ & $-175.3(-4.7)$ & $-181.9(1.9)$ & $-177.5(-2.5)$ & $-169.9(-10.1)$ & $-174.9(-5.1)$ & $-173.5(-6.5)$ & $\begin{array}{c}\mathbf{- 1 8 0 . 0}[\mathbf{2 0}]^{\prime} \\
-178.20^{v}\end{array}$ & $-176.3 \pm 3.3$ & -3.7 \\
\hline 26 & $\mathrm{CFI}_{2} \mathrm{Cl}$ & $-70.1(0.1)$ & $-69.8(-0.2)$ & $-71.6(1.6)$ & $-57.9(-12.1)$ & $-50.4(-19.6)$ & $-58.8(-11.2)$ & $\begin{array}{c}-70[30]^{\prime}, \\
-79.49^{v}\end{array}$ & $-60.5 \pm 7.4$ & -9.5 \\
\hline 27 & $\mathrm{CFBr}_{2} \mathrm{I}$ & & $-71.3(1.3)$ & & $-68.6(-1.4)$ & $-84.1(14.1)$ & $-72.9(2.9)$ & $-70[25]^{\prime}, 79.41^{v}$ & $-72.3 \pm 7.2$ & 2.3 \\
\hline
\end{tabular}


$\left(\Delta \mathrm{H}_{\mathrm{f}}^{\circ}\right)_{\mathrm{m}}(\mathrm{g}) \mathrm{kJ}^{\mathrm{m}} \mathrm{mol}^{-1}(298.15 \mathrm{~K})$

\begin{tabular}{|c|c|c|c|c|c|c|c|c|c|}
\hline \multirow[t]{2}{*}{ Species } & \multicolumn{3}{|c|}{ DFT/SPK-ATZP } & \multicolumn{3}{|c|}{ MP2/SPK-ATZP } & \multirow[b]{2}{*}{$\begin{array}{c}\text { Experim. } \\
(E X)^{b}\end{array}$} & \multirow[b]{2}{*}{$\begin{array}{l}\text { Eval. } \\
(\mathrm{EV})^{b}\end{array}$} & \multirow[b]{2}{*}{ EX-EV } \\
\hline & $\mathbf{R 1}^{a, c}$ & $\mathbf{R} \mathbf{2}^{\mathbf{a}, c}$ & $\mathbf{R 3}^{a, c}$ & $\mathbf{R} \mathbf{1}^{a, c}$ & $\mathbf{R} \mathbf{2}^{a, c}$ & $\mathbf{R 3}^{\mathbf{a}, c}$ & & & \\
\hline $\begin{array}{c}\text { Mean Abs. } \\
\text { Error }^{\times 1} \text { (MAE) }\end{array}$ & $(5.8)$ & (4.3) & $(4.5)$ & (5.1) & (3.9) & (4.1) & [11.8] & [3.2] & $(2.2)$ \\
\hline $\begin{array}{l}\text { Mean Abs. } \\
\text { Error (corr.) } \\
\text { (MAEC) }\end{array}$ & $(5.8)$ & (4.3) & (4.5) & $(3.9)^{x 2}$ & $(2.8)^{x 2}$ & $(3.0)^{x^{2}}$ & {$[9.5]^{x 2}$} & {$[3.0]^{x 2}$} & $(1.7)^{x 2}$ \\
\hline rms error ${ }^{\times 3}$ & 7.9 & 5.8 & 5.7 & 5.1 & 4.5 & 4.1 & 16.1 & 3.6 & 2.1 \\
\hline $\begin{array}{c}\text { Mean Signed } \\
\text { Error from } \\
(E X)^{\times 4} \text { (MSE) }\end{array}$ & -4.4 & -0.5 & -1.5 & -2.3 & -1.4 & -1.3 & & & -0.3 \\
\hline
\end{tabular}

${ }^{a}$ R1, R2, R3 refer to reactions (1), (2) and (3) ${ }^{b}$ Values in brackets represent reported experimental uncertainties of $\left(\Delta \mathrm{Hf}^{\circ}\right)_{\mathrm{m}}(\mathrm{g})$. The listed uncertainties of evaluations correspond to estimated $95 \%$ confidence limits as customary in thermochemistry. The most reliable values of $\left(\Delta \mathrm{Hf}{ }^{\circ}\right)_{\mathrm{m}}(\mathrm{g})$ are written in bold. ${ }^{c}$ Values in parentheses represent errors (deviations) from experimental data $\left[\left(\Delta \mathrm{H} \mathrm{f}^{\circ}\right)_{\mathrm{m}}(\mathrm{g})\right.$ experimental $-\left(\Delta \mathrm{H} \mathrm{f}^{\circ}\right)_{\mathrm{m}}(\mathrm{g})$ computed]. ${ }^{d}$ From ref. 44, ${ }^{e}$ From ref. $45,{ }^{f}$ From ref. $43,{ }^{g}$ From ref. 11, ${ }^{h}$ From ref. 49, ${ }^{i}$ From ref. 50, ${ }^{k}$ From ref. 46, ' From ref. $15,{ }^{m}$ From ref. 10, ${ }^{n}$ From ref. 51, ${ }^{o}$ From ref. 52, ${ }^{p}$ From ref. 53, ${ }^{q}$ From ref. 54, ${ }^{\prime}$ From ref. 55, ${ }^{s}$ From ref. 56, ${ }^{t}$ From ref. 57, ${ }^{u}$ From ref. 58, ${ }^{v}$ From ref. 14 (according to the authors the estimated error is less than $\pm 8.4 \mathrm{~kJ} \mathrm{~mol}^{-1}$ except in compounds containing $\mathrm{ClI}$ and $\mathrm{BrI}$ bonds where it may be as high as $\left.\pm 20.9 \mathrm{~kJ} \mathrm{~mol}^{-1}\right)$, ${ }^{w}$ From ref. 59, ${ }^{x}{ }_{o}$ Evaluations from ref. $47^{x 1}$ Mean Absolute Error $\mathrm{I}\left(\Delta \mathrm{H}^{\circ}\right)_{\mathrm{m}}(\mathrm{g})$ experimental $-\left(\Delta \mathrm{H} \mathrm{f}^{\circ}\right)_{\mathrm{m}}(\mathrm{g})$ computed ${ }^{x 2}$ Mean Absolute Error $\mathrm{I}\left(\Delta \mathrm{H} \mathrm{f}^{\circ}\right)_{\mathrm{m}}(\mathrm{g})$ experimental $-\left(\Delta \mathrm{H} \mathrm{f}^{\circ}\right)_{\mathrm{m}}(\mathrm{g})$

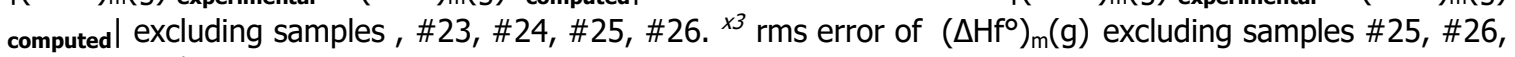
\#27, \#28. ${ }^{x 4}$ Mean Signed Error $\left[\left(\Delta H f^{\circ}\right)_{m}(g)\right.$ experimental $\left.-\left(\Delta H f^{\circ}\right)_{m}(g)_{\text {computed }}\right]$.

TABLE 1B: $\left(\Delta \mathrm{Hf}^{\circ}\right)_{\mathrm{m}}(\mathrm{g})$ values of halomethanes, experimental and computed at CCSD(T)/SPK-ATZP level of theory.

\begin{tabular}{|c|c|c|c|c|c|c|c|}
\hline & & \multicolumn{6}{|c|}{$\left(\Delta \mathrm{H}_{\mathrm{f}}^{\circ}\right)_{\mathrm{m}}(\mathrm{g}) \mathrm{kJ}_{\mathrm{mol}} \mathrm{mo}^{-1}(298.15 \mathrm{~K})$} \\
\hline & \multirow[t]{2}{*}{ Species } & \multicolumn{3}{|c|}{ CCSD(T)/SPK-ATZP } & \multirow[b]{2}{*}{$\begin{array}{c}\text { Experim. } \\
(E X)^{b}\end{array}$} & \multirow[b]{2}{*}{$\begin{array}{l}\text { Eval. } \\
\text { (EV) }^{b}\end{array}$} & \multirow[b]{2}{*}{ EX-EV } \\
\hline & & $\mathbf{R 1}^{a, c}$ & $\mathbf{R} 2^{a, c}$ & $\mathbf{R 3}^{a, c}$ & & & \\
\hline 1 & $\mathrm{CHBr}_{3}$ & $51.0(-2.9)$ & $47.5(0.6)$ & & $\begin{array}{c}\mathbf{4 8 . 1 0}[\mathbf{1 . 3}]^{f}, \\
55.1[4.9]^{d}, 55.4 \\
{[3.3]^{e}, 23.8[4.5]^{g}}\end{array}$ & $47.4 \pm 2.6$ & 0.7 \\
\hline 2 & $\mathrm{CH}_{3} \mathrm{Br}$ & $-35.2(-2.5)$ & $-31.9(-5.8)$ & & $\begin{array}{c}\mathbf{- 3 7 . 7}[\mathbf{1 . 5}]^{h}, \\
-35.4[1.1]^{g}, \\
-35.76^{f},-38.1[1.3]^{i}\end{array}$ & $-35.5 \pm 6.3$ & -2.2 \\
\hline 3 & $\mathrm{CH}_{2} \mathrm{Br}_{2}$ & $1.4(1.8)$ & $9.0(-5.8)$ & & $\begin{array}{c}\mathbf{3 . 2} \text { [3.4 }^{\boldsymbol{k}}{ }^{\prime} \\
3.5^{f}, 10.0[15]^{\prime}\end{array}$ & $3.6 \pm 6.5$ & -0.4 \\
\hline
\end{tabular}




\section{$\left(\Delta \mathrm{H}_{\mathrm{f}}{ }^{\circ}\right)_{\mathrm{m}}(\mathrm{g}){\mathrm{kJ} . \mathrm{mol}^{-1}(298.15 \mathrm{~K})}$}

\begin{tabular}{|c|c|c|c|c|c|c|c|}
\hline & & & $(L$ & $\left.\mathrm{t}_{\mathrm{f}}{ }^{\circ}\right) \mathrm{m}(\mathrm{g}) \mathrm{kJ} . \mathrm{m}$ & $1 \mathrm{OI}^{-1}(298.15 \mathrm{~K})$ & & \\
\hline & Species & & $\mathrm{D}(\mathrm{T}) / \mathrm{SPK}-\mathrm{AT}$ & & & & \\
\hline & & $\mathbf{R 1}^{a, c}$ & $\mathbf{R} \mathbf{2}^{a, c}$ & $\mathbf{R 3}^{a, c}$ & $\begin{array}{l}\text { Experim. } \\
(\mathrm{EX})^{b}\end{array}$ & $\begin{array}{l}\text { Eval. } \\
(E V)^{b}\end{array}$ & EX-EV \\
\hline 4 & $\mathrm{CHCl}_{3}$ & $-99.0(-3.9)$ & $-101.7(-1.2)$ & & $\begin{array}{l}\mathbf{- 1 0 2 . 9}[\mathbf{2 . 5}]^{\mathbf{m}} \\
-103.2^{n},-103.39^{f}\end{array}$ & $-98.0 \pm 4.6$ & -4.9 \\
\hline 5 & $\mathrm{CH}_{3} \mathrm{Cl}$ & $-83.2(1.3)$ & $-83.1(1.2)$ & & $\begin{array}{c}\mathbf{- 8 1 . 9}[\mathbf{1 . 5}]^{\mathbf{m}} \\
-81.9[0.5]^{k}{ }^{\prime} \\
-82.18^{f}\end{array}$ & $-82.9 \pm 0.4$ & 1.0 \\
\hline 6 & $\mathrm{CH}_{2} \mathrm{Cl}_{2}$ & & $-96.9(1.2)$ & & $\begin{array}{c}\mathbf{- 9 5 . 7}\left[\mathbf{1 . 3} \mathbf{3}^{\mathbf{g , k}},{ }^{\prime}{ }^{-95.1}[2.5]^{m, n},\right. \\
-95.4[1.1]^{g}\end{array}$ & $-96.6 \pm 0.5$ & 0.9 \\
\hline 7 & $\mathrm{CH}_{2} \mathrm{BrCl}$ & $-44.9(0.8)$ & $-46.3(2.2)$ & & $\begin{array}{c}-44.1\left[^{[1.9]^{k}}{ }^{\prime}\right. \\
-20[7]^{m},-43.3^{f} \\
-45.0[5]^{\prime}\end{array}$ & $-45.9 \pm 0.9$ & 1.8 \\
\hline 8 & $\mathrm{CHCl}_{2} \mathrm{~F}$ & & $-283.2(-0.1)$ & $-279.6(-3.7)$ & $\begin{array}{l}\mathbf{- 2 8 3 . 3}[\mathbf{1 3}]^{p, q} \\
-284.20^{f},-284.1^{\prime r}\end{array}$ & $-282.8 \pm 1.2$ & -0.5 \\
\hline 9 & $\mathrm{CHCl}_{2} \mathrm{Br}$ & $-49.5(-0.6)$ & $-52.2(2.1)$ & $-48.7(-1.4)$ & $\begin{array}{c}-\mathbf{5 0 . 1}[\mathbf{1 . 8}]^{d}{ }^{\prime} \\
-50.3^{f},-50.9[2.4]^{x o}\end{array}$ & $-48.2 \pm 3.2$ & -1.9 \\
\hline 10 & $\mathrm{CHBr}_{2} \mathrm{Cl}$ & $-0.6(0.6)$ & $1.1(-1.1)$ & $0.1(-0.1)$ & $\begin{array}{l}0.0\left[3.4^{] f}\right. \\
4.1^{d}, 10.0^{\prime}\end{array}$ & $-0.8 \pm 1.0$ & 0.8 \\
\hline 11 & $\mathrm{CFCl}_{3}$ & $-286.7(-2.0)$ & $-287.8(-0.9)$ & $-289.4(0.7)$ & $\begin{array}{l}-288.7[6.3]^{p} \\
-290.1^{f},-278^{s^{\prime}}\end{array}$ & $-287.7 \pm 1.3$ & -1 \\
\hline 12 & $\mathrm{CF}_{3} \mathrm{Br}$ & $-649.9(1.0)$ & $-649.0(0.1)$ & $-654.4(5.5)$ & $\begin{array}{l}\mathbf{- 6 4 8 . 9}[2.9]^{p} \\
-647.0^{t},-651.57^{f}\end{array}$ & $-648.3 \pm 5.0$ & -0.6 \\
\hline 13 & $\mathrm{CF}_{2} \mathrm{Cl}_{2}$ & $-492.4(0.8)$ & $-495.1(3.5)$ & $-495.2(3.6)$ & 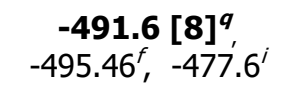 & $-491.5 \pm 1.2$ & -0.1 \\
\hline 14 & $\mathrm{CHF}_{2} \mathrm{Cl}$ & $-480.8(-0.8)$ & $-483.3(1.7)$ & $-479.9(-1.7)$ & $\begin{array}{c}-481.6[13]^{q} \\
-482.3^{u},-482.60^{f}\end{array}$ & $-480.1 \pm 3.4$ & -1.5 \\
\hline 15 & $\mathrm{CH}_{2} \mathrm{ClF}$ & $-263.3(1.4)$ & $-264.2(2.3)$ & & $\begin{array}{c}\mathbf{- 2 6 1 . 9}[\mathbf{1 3}]^{q} \\
-263.60^{f}\end{array}$ & $-264.3 \pm 1.9$ & 2.4 \\
\hline 16 & $\mathrm{CF}_{3} \mathrm{Cl}$ & $-707.1(-2.9)$ & $-710.9(0.9)$ & $-711.6(1.6)$ & $\begin{array}{c}\mathbf{- 7 1 0 . 0}[\mathbf{3 . 3}]^{q} \\
-710.08^{f},-707.3^{w}\end{array}$ & $-708.9 \pm 3.5$ & -1.1 \\
\hline 17 & $\mathrm{CH}_{2} \mathrm{ICl}$ & $12.0(-1.3)$ & $10.6(0.1)$ & & $10.7[1.9]^{k}, 13.6^{v}$ & $9.6 \pm 2.9$ & 1.1 \\
\hline 18 & $\mathrm{CH}_{2} \mathrm{IBr}$ & $57.4(-2.4)$ & $55.7(-0.7)$ & & $55.0[3.4]^{k}, 57.1^{v}$ & $58.0 \pm 2.3$ & -3.0 \\
\hline
\end{tabular}




\begin{tabular}{|c|c|c|c|c|c|c|c|}
\hline & & & $(\Delta$ & $\left.\mathrm{t}_{\mathrm{f}}^{\circ}\right)_{\mathrm{m}}(\mathrm{g}) \mathrm{kJ} . \mathrm{n}$ & $\mathrm{ol}^{-1}(298.15 \mathrm{~K})$ & & \\
\hline & Species & & SD(T)/SPK-AT & & & & \\
\hline & & $\mathbf{R} \mathbf{1}^{a, c}$ & $\mathbf{R} \mathbf{2}^{a, c}$ & $\mathbf{R} 3^{a, c}$ & $\begin{array}{l}\text { Experim. } \\
(E X)^{b}\end{array}$ & $\begin{array}{l}\text { Eval. } \\
(E V)^{b}\end{array}$ & EX-EV \\
\hline 19 & $\mathrm{CHI}_{2} \mathrm{Cl}$ & $114.7(-4.7)$ & $113.3(-3.3)$ & & $\begin{array}{c}\mathbf{1 1 0 . 0}[\mathbf{3 0}]^{\prime} \\
112.9^{v}\end{array}$ & $113.5 \pm 2.3$ & -3.5 \\
\hline 20 & $\mathrm{CHI}_{2} \mathrm{Br}$ & $160.6(4.4)$ & $167.0(-2.0)$ & & $\begin{array}{c}\mathbf{1 6 5 . 0}[\mathbf{3 5}]^{\prime}, \\
158.20^{\prime}\end{array}$ & $165.4 \pm 4.7$ & -0.4 \\
\hline 21 & $\mathrm{CHCl}_{2} \mathrm{I}$ & $9.8(-4.8)$ & $7.1(-2.1)$ & $7.9(-2.9)$ & $5.0[20]^{\prime}, 8.28^{v}$ & $7.0 \pm 2.3$ & -2.0 \\
\hline 22 & $\mathrm{CHBr}_{2} \mathrm{I}$ & $104.0(6.0)$ & $107.2(2.8)$ & $101.6(8.4)$ & $\begin{array}{c}\mathbf{1 1 0 . 0}[\mathbf{2 5}]^{\prime} \\
93.51^{v}\end{array}$ & $105.6 \pm 4.0$ & 4.4 \\
\hline 23 & $\mathrm{CF}_{2} \mathrm{ClI}$ & $-375.8(-4.2)$ & $-379.7(-0.3)$ & $-377.0(-3.0)$ & $\begin{array}{c}\mathbf{- 3 8 0 . 0}[\mathbf{2 5}]^{\prime} \\
-379.07^{v}\end{array}$ & $-372.3 \pm 1.3$ & -7.7 \\
\hline 24 & $\mathrm{CF}_{2} \mathrm{BrI}$ & & & & $\begin{array}{c}-\mathbf{3 2 5 . 0}[\mathbf{2 5}]^{\prime} \\
-324.30^{v}\end{array}$ & $-325.1 \pm 6.0$ & 0.1 \\
\hline 25 & $\mathrm{CFCl}_{2} \mathrm{I}$ & $-173.7(-6.3)$ & $-179.6(-0.4)$ & $-178.3(-1.7)$ & $\begin{array}{c}\mathbf{- 1 8 0 . 0}[\mathbf{2 0}]^{\prime} \\
.178 .20^{v}\end{array}$ & $-176.3 \pm 3.3$ & -3.7 \\
\hline 26 & $\mathrm{CFI}_{2} \mathrm{Cl}$ & $-59.8(-10.2)$ & $-52.5(-17.5)$ & $-59.0(-11.0)$ & $\begin{array}{c}-70[30]^{\prime}, \\
-79.49^{v}\end{array}$ & $-60.5 \pm 7.4$ & -9.5 \\
\hline 27 & $\mathrm{CFBr}_{2} \mathrm{I}$ & $-69.8(-0.2)$ & $-81.8(11.8)$ & $-64.3(-5.7)$ & $-70[25]^{\prime},-79.41^{v}$ & $-72.3 \pm 7.2$ & 2.3 \\
\hline & $\begin{array}{l}\text { an Abs. } \\
x^{x 1} \text { (MAE) }\end{array}$ & (2.8) & (2.8) & (3.6) & & [3.2] & $(2.2)$ \\
\hline & $\begin{array}{l}\text { an Abs. } \\
\text { r (corr.) } \\
\text { MAEC) }\end{array}$ & $(2.2)^{x 2}$ & $(2.3)^{x 2}$ & $(3.2)^{x 2}$ & & {$[3.0]^{x 2}$} & $(1.7)^{x^{2}}$ \\
\hline & error ${ }^{\times 3}$ & 2.8 & 3.4 & 4.0 & & 3.6 & 2.1 \\
\hline & $\begin{array}{l}\text { n Signed } \\
\text { or from } \\
x 4 \text { (MSE) }\end{array}$ & -1.3 & -0.4 & -0.8 & & & -0.3 \\
\hline $\begin{array}{l}{ }^{a} \mathrm{R} 1 \\
\text { unce } \\
\text { conf } \\
\text { bold } \\
\left(\Delta \mathrm{H}_{\mathrm{f}}\right. \\
50, \\
\text { ref. } \\
\text { auth } \\
\text { whe } \\
\text { Erro } \\
(\Delta \mathrm{H} \\
\# 25\end{array}$ & $\begin{array}{l}\mathrm{R} 2, \mathrm{R} 3 \text { re } \\
\text { tainties of } \\
\text { lence limits } \\
{ }^{c} \text { Values in } \\
)_{\mathrm{m}}(\mathrm{g})_{\text {computed }} \\
\text { From ref. } \\
4{ }^{\prime}{ }^{\prime} \text { From } \\
\text { is the estin } \\
\text { it may be } \\
\mid\left(\Delta \mathrm{Hf}^{\circ}\right)_{\mathrm{m}}( \\
)_{\mathrm{m}}(\mathrm{g}) \text { compu } \\
\# 26, \# 27 \text {, }\end{array}$ & $\begin{array}{l}\text { er to reactions } \\
\left(\Delta \mathrm{Hf}^{\circ}\right) \mathrm{m}(\mathrm{g}) . \mathrm{TH} \\
\text { as customary ir } \\
\text { parentheses re } \\
{ }^{d} \text { From ref. } 44, \\
6,{ }^{6} \text { From ref. } 1 \\
\text { ef. } 55,{ }^{s} \text { From } \\
\text { ated error is les } \\
\text { as high as } \pm 20 . c \\
{ }^{\text {) experimental }}- \\
\text { ed } \text { excluding sa } \\
\text { 28. }{ }^{\times 4} \text { Mean Sic }\end{array}$ & 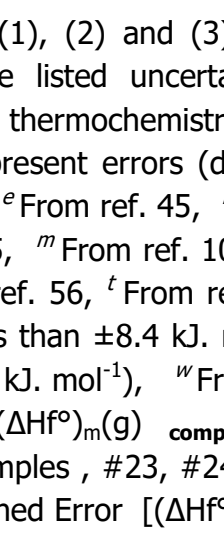 & 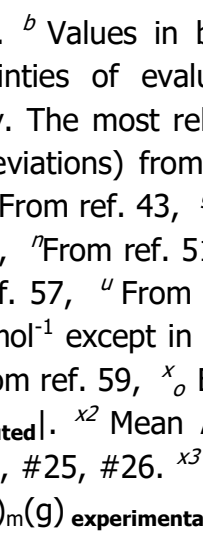 & $\begin{array}{l}\text { ackets represent re } \\
\text { ations correspond } \\
\text { able values of }\left(\Delta \mathrm{Hf}^{\circ}\right) \\
\text { experimental data } \\
\text { From ref. } 11,{ }^{h} \text { From } \\
{ }^{\circ} \text { From ref. } 52,{ }^{p} \mathrm{~F} \\
\text { ef. } 58,{ }^{v} \text { From ref. } 1 \\
\text { ompounds containing } \\
\text { valuations from ref. } 4 \\
\text { bsolute Error } \mathrm{l}(\Delta \mathrm{Hf} \\
\text { ms error }\left(\Delta \mathrm{H} f^{\circ}\right) \mathrm{m}(g \\
-\left(\Delta \mathrm{Hf}^{\circ}\right)_{\mathrm{m}}(\mathrm{g}) \text { computed }\end{array}$ & $\begin{array}{l}\text { orted experim } \\
0 \text { estimated } \\
\mathrm{n}(\mathrm{g}) \text { are writte } \\
\left.\mathrm{H}_{\mathrm{f}}^{\circ}\right)_{\mathrm{m}}(\mathrm{g})_{\text {experime }} \\
\text { ef. } 49,{ }^{i} \text { From } \\
\mathrm{m} \text { ref. } 53,{ }^{q} \\
\text { (according to } \\
\text { CII and BrI b } \\
{ }^{x 1} \text { Mean Abs } \\
\text { ) }_{\mathrm{m}}(\mathrm{g}) \text { experimen } \\
\text { excluding sam }\end{array}$ & \\
\hline
\end{tabular}


studied $\mid\left(\Delta \mathrm{H}_{\mathrm{f}}{ }^{\circ}\right)_{\mathrm{m}}(\mathrm{g})_{\text {experimental }}-\left(\Delta \mathrm{H}_{\mathrm{f}}{ }^{\circ}\right)_{\mathrm{m}}(\mathrm{g})_{\text {computed }} \mathrm{l}$ is near or below $4.5 \mathrm{~kJ} \cdot \mathrm{mol}^{-1}$. This value is very similar to the mean absolute deviation of $\sim 4.184 \mathrm{~kJ} \cdot \mathrm{mol}^{-1}$ accuracy found for a large number of molecules with reliable experimental standard molar enthalpies of formation. Exceptions are observed at the DFT and MP2 level when reaction R1 was used $(\mathrm{MAE}=5.8$ and 5.1 respectively, Table $1 \mathrm{~A})$.

Computations at the DFT level which are computationally inexpensive gave MAE values that range from 4.3 to $5.8 \mathrm{~kJ} \mathrm{~mol}^{-1}$. Computations at the MP2 level gave MAE values that range from 4.1 to $5.1 \mathrm{~kJ} \mathrm{~mol}^{-1}$ while those at the $\operatorname{CCSD}(\mathrm{T})$ level range from 2.8 to 3.6 kJ. mol ${ }^{-1}$ (Table 1A \& 1B). Deviations (MAE) between experimental and computed values decrease if the iodine containing mixed halomethanes $\mathrm{CF}_{2} \mathrm{Cl}, \mathrm{CF}_{2} \mathrm{BrI}_{2} \mathrm{CFCl}_{2} \mathrm{I}$ and $\mathrm{CFI}_{2} \mathrm{Cl}$ are excluded since they tend to rise with increasing halogen substitution. ${ }^{54}$ In such a ca-

se, when the above halomethanes are excluded MAE of the compounds studied ranges from 2.8 to $3.9 \mathrm{~kJ} \mathrm{~mol}^{-1}$ at the MP2 and from 2.2 to $3.2 \mathrm{~kJ} \cdot \mathrm{mol}^{-1}$ at the $\operatorname{CCSD}(\mathrm{T})$ level respectively while it remains unaffected at the DFT level (Table $1 A \& 1 B$ ) .

Furthermore, the small mean signed errors (MSE) between experimental and computed values ranging from -0.4 to $-2.3 \mathrm{~kJ} \cdot \mathrm{mol}^{-1}$ (except at the DFT level when reaction R1 was used, MSE $=-4.4$ ) show that the computed standard molar enthalpies of formation $\left(\Delta \mathrm{H}_{\mathrm{f}}{ }^{\circ}\right)_{\mathrm{m}}(\mathrm{g})$ as an average lie in most cases slightly higher than the corresponding reported experimental values (Table $1 \mathrm{~A} \& 1 \mathrm{~B}$ ).

The rms errors of computed values using the DFT, MP2 and $\operatorname{CCSD}(\mathrm{T})$ methods range

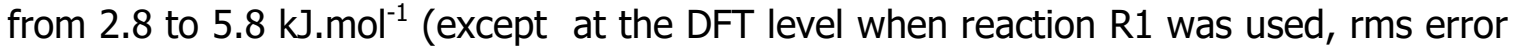
$=7.9$ ) which are well below the rms experimental uncertainty of $16.1 \mathrm{~kJ}^{\mathrm{mol}}{ }^{-1}$ (Table $1 \mathrm{~A}$ $\& 1 B)$.

Plots of $\left(\Delta \mathrm{H}_{\mathrm{f}}{ }^{\circ}\right)_{\mathrm{m}}(\mathrm{g})_{\text {experimental }}$ versus $\left(\Delta \mathrm{H}_{\mathrm{f}}{ }^{\circ}\right)_{\mathrm{m}}(\mathrm{g})_{\text {computed }}$ values using reactions (R1)-(R3) at the DFT, MP2 and $\operatorname{CCSD}(T)$ levels of theory show excellent correlation and the following best-fitted linear equations and $\mathrm{R}^{2}$ values are obtained:

$$
\begin{aligned}
& \left(\Delta \mathrm{H}_{\mathrm{f}}{ }^{\circ}\right)_{\mathrm{m}(\text { experimental })}=1.0210 *\left(\Delta \mathrm{H}_{\mathrm{f}}{ }^{\circ}\right)_{\mathrm{m}(\mathrm{DFT}, \text { reaction R1 })}-2.9580 ; \quad \mathrm{R}^{2}=0.9990 \\
& \left(\Delta \mathrm{H}_{\mathrm{f}}{ }^{\circ}\right)_{\mathrm{m}(\text { experimental })}=1.0114 *\left(\Delta \mathrm{H}_{\mathrm{f}}{ }^{\circ}\right)_{\mathrm{m}(\mathrm{DFT}, \text { reaction R2) }}+0.4942 ; \quad \mathrm{R}^{2}=0.9989 \\
& \left(\Delta \mathrm{H}_{\mathrm{f}}{ }^{\circ}\right)_{\mathrm{m}(\text { experimental })}=0.9998 *\left(\Delta \mathrm{H}_{\mathrm{f}}{ }^{\circ}\right)_{\mathrm{m}(\mathrm{DFT}, \text { reaction R3) }}-1.1264 ; \quad \mathrm{R}^{2}=0.9996 \\
& \left(\Delta \mathrm{H}_{\mathrm{f}}{ }^{\circ}\right)_{\mathrm{m}(\text { experimental })}=1.0227 *\left(\Delta \mathrm{H}_{\mathrm{f}}{ }^{\circ}\right)_{\mathrm{m}(\mathrm{MP} 2 \text {, reaction R1) })}+1.7667 ; \quad \mathrm{R}^{2}=0.9996 \\
& \left(\Delta \mathrm{H}_{\mathrm{f}}^{\circ}\right)_{\mathrm{m}(\text { experimental })}=1.0053 *\left(\Delta \mathrm{H}_{\mathrm{f}}^{\circ}\right)_{\mathrm{m}(\mathrm{MP2}, \text { reaction R2 })}+0.4192 ; \quad \mathrm{R}^{2}=0.9994 \\
& \left(\Delta \mathrm{H}_{\mathrm{f}}{ }^{\circ}\right)_{\mathrm{m}(\text { experimental })}=1.0069 *\left(\Delta \mathrm{H}_{\mathrm{f}}{ }^{\circ}\right)_{\mathrm{m}(\mathrm{MP2}, \text { reaction R3 })}+0.5835 ; \quad \mathrm{R}^{2}=0.9996 \\
& \left(\Delta \mathrm{H}_{\mathrm{f}}{ }^{\circ}\right)_{\mathrm{m}(\text { experimental })}=1.0018 *\left(\Delta \mathrm{H}_{\mathrm{f}}{ }^{\circ}\right)_{\mathrm{m}(\mathrm{CCSD}(\mathrm{T}) \text {, reaction } \mathrm{R} 1)}+0.5885 ; \mathrm{R}^{2}=0.9991
\end{aligned}
$$




$$
\begin{aligned}
& \left(\Delta \mathrm{H}_{\mathrm{f}}^{\circ}\right)_{\mathrm{m} \text { (experimental) }}=0.9957 *\left(\Delta \mathrm{H}_{\mathrm{f}}{ }^{\circ}\right)_{\mathrm{m}(\mathrm{CCSD}(\mathrm{T}) \text {, reaction R2) }}-0.2123 ; \mathrm{R}^{2}=0.9991 \\
& \left(\Delta \mathrm{H}_{\mathrm{f}}^{\circ}\right)_{\mathrm{m} \text { (experimental) }}=0.9948 *\left(\Delta \mathrm{H}_{\mathrm{f}}{ }^{\circ}\right)_{\mathrm{m}(\mathrm{CCSD}(\mathrm{T}) \text {, reaction } \mathrm{R} 3))}-0.8595 ; \mathrm{R}^{2}=0.9995
\end{aligned}
$$

Figure 1 - Figure 3 show the graphs obtained at the DFT, MP2 and $\operatorname{CCSD}(\mathrm{T})$ levels using reaction R1. The corresponding linear equations are (1), (4) and (7) with $R^{2}$ values $0.9990,0.9996$ and 0.9991 respectively.

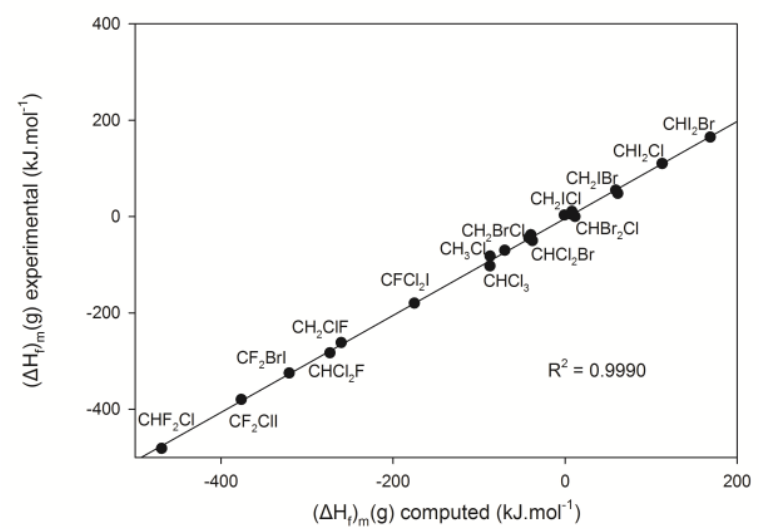

Figure 1. $\left(\Delta \mathrm{H}_{\mathrm{f}}{ }^{\circ}\right)_{\mathrm{m}}(\mathrm{g})$ of halomethane molecules in Table $1 \mathrm{~A}$ obtained at the DFT level of theory and ADZP basis set using reaction $\mathrm{R} 1$ versus their corresponding experimental values.

The reliable $\left(\Delta \mathrm{H}_{\mathrm{f}}{ }^{\circ}\right)_{\mathrm{m}}(\mathrm{g})$ average values of the compounds listed in Table $1 \mathrm{~A} \& 1 \mathrm{~B}$ [column: Evaluations $(\mathrm{EV})]$ were evaluated by extrapolating their computed values, which are obtained in this work, from correlation equations (1) - (9) and isodesmic reactions R1 - R3. The reported uncertainties correspond to estimated $95 \%$ confidence limits as customary in thermochemistry.

The reliable $\left(\Delta \mathrm{H}_{\mathrm{f}}{ }^{\circ}\right)_{\mathrm{m}}(\mathrm{g})$ average values of the environmentally important $\mathrm{CHCl}_{2} \mathrm{Br}$ and $\mathrm{CHBr}_{2} \mathrm{Cl}$ were evaluated as described above. Both species have been considered important sources of reactive halogens in the atmosphere and reliable $\left(\Delta \mathrm{H}_{\mathrm{f}}{ }^{\circ}\right)_{\mathrm{m}}(\mathrm{g})$ experimental data ${ }^{\mathbf{4 6}}$ were not available until recently.

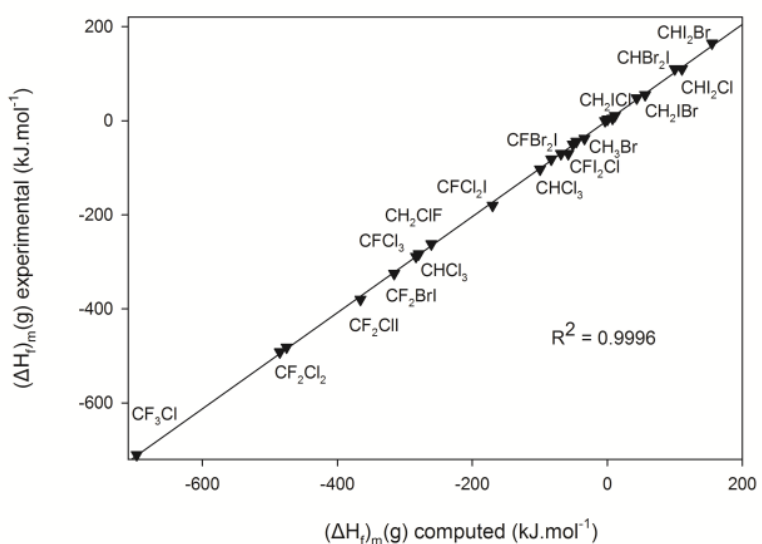

Figure 2. $\left(\Delta H f^{\circ}\right) \mathrm{m}(\mathrm{g})$ of halomethane molecules in Table $1 \mathrm{~A}$ obtained at the MP2 level of theory and SPK-ATZP basis set using reaction R1 vs. their corresponding experimental values.

The $\left(\Delta \mathrm{H}_{\mathrm{f}}^{\circ}\right)_{\mathrm{m}}(\mathrm{g})$ average value of $\mathrm{CHBr}_{2} \mathrm{Cl}$ was found to be $-0.8 \pm 1.0 \mathrm{~kJ}^{\mathrm{mol}}{ }^{-1}$ in excellent agreement with the value reported by the Argonne Thermochemical Network ${ }^{43} 0.0 \pm 3.4$ $\mathrm{kJ} \cdot \mathrm{mol}^{-1}$. The experimental value reported by Shuman et al. ${ }^{44}$ was $4.1 \pm 4.9 \mathrm{~kJ} . \mathrm{mol}^{-1}$. The standard molar enthalpy of formation of $\mathrm{CHBr}_{2} \mathrm{Cl}$ has also been estimated by Gurvich et al. ${ }^{15}$ to be $10 \mathrm{~kJ} \cdot \mathrm{mol}^{-1}$, at $298 \mathrm{~K}$, however with uncertainty greater than $10 \mathrm{~kJ} \cdot \mathrm{mol}^{-1}$.

Similarly, the reliable $\left(\Delta \mathrm{H}_{\mathrm{f}}{ }^{0}\right)_{\mathrm{m}}(\mathrm{g})$ average value of $\mathrm{CHCl}_{2} \mathrm{Br}$ was found to be $-48.2 \pm 3.2 \mathrm{~kJ} \cdot \mathrm{mol}^{-1}$ in the limits of the estimated uncertainty of the experimental value reported by Shuman et al. ${ }^{44}$ $50.1 \pm 1.8 \mathrm{~kJ} \cdot \mathrm{mol}^{-1}$. The estimated value reported by Davalos et al. ${ }^{47}$ was $-50.9 \pm 2.4$ $\mathrm{kJ} \cdot \mathrm{mol}^{-1}$ and by the Argonne Thermochemical Network $^{43}-50.3 \pm 1.3 \mathrm{~kJ} \cdot \mathrm{mol}^{-1}$. 


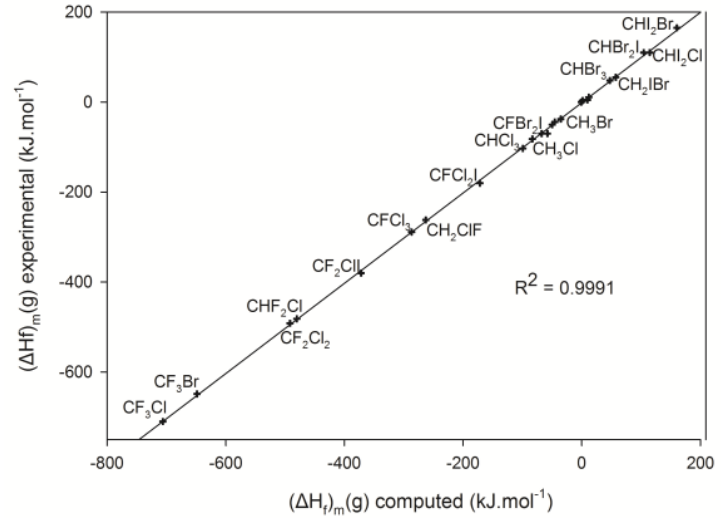

Figure 3. $\left(\Delta \mathrm{H}_{\mathrm{f}}{ }^{\circ}\right)_{\mathrm{m}}(\mathrm{g})$ of halomethane molecules in Table 1B obtained at the $\operatorname{CCSD}(T)$ level of theory and SPK- ATZP basis set using reaction R1 vs. their corresponding experimental values.

The reliable $\left(\Delta \mathrm{Hf}^{\circ}\right) \mathrm{m}(\mathrm{g})$ average value of $\mathrm{CH} 2 \mathrm{BrCl}$ was also determined in this study and found to be equal to $-45.9 \pm 0.9 \mathrm{~kJ} . \mathrm{mol}-1$ which is in agreement within the estimated uncertainty with the experimental value reported by Lago et al46 $-44.1 \pm 1.9 \mathrm{~kJ} . \mathrm{mol}-1$. The value reported by the Argonne Thermochemical Network43 is $-43.3 \pm 1.4 \mathrm{~kJ} . \mathrm{mol}-1$.

The $\left(\Delta \mathrm{H}_{\mathrm{f}}{ }^{\circ}\right)_{\mathrm{m}}(\mathrm{g})$ average values of a series of chlorofluoromethanes and one bromofluoromethane $\left(\mathrm{CHCl}_{2} \mathrm{~F}, \mathrm{CFCl}_{3}, \mathrm{CF}_{2} \mathrm{Cl}_{2}, \mathrm{CHF}_{2} \mathrm{Cl}, \mathrm{CH}_{2} \mathrm{ClF}, \mathrm{CF}_{3} \mathrm{Cl}, \mathrm{CF}_{3} \mathrm{Br}\right)$ were evaluated and found in close agreement with the corresponding experimental literature values (Table $1 \mathrm{~A}$ $\&$ 1B). The $\left[\left(\Delta \mathrm{H}_{\mathrm{f}}{ }^{\circ}\right)_{\mathrm{m}}(\mathrm{g})_{\text {experimental }}-\left(\Delta \mathrm{H}_{\mathrm{f}}{ }^{\circ}\right)_{\mathrm{m}}(\mathrm{g})_{\text {evaluated }}\right]$ standard molar enthalpy of formation differences lie between $-1.5-2.4 \mathrm{~kJ} \cdot \mathrm{mol}^{-1}$.

The $\left(\Delta \mathrm{H}_{\mathrm{f}}{ }^{\circ}\right)_{\mathrm{m}}(\mathrm{g})$ average values of the iodine containing dihalomethanes $\mathrm{CH}_{2} \mathrm{ICl}$ and $\mathrm{CH}_{2} \mathrm{IBr}$ were also estimated and found to be $9.6 \pm 2.9 \mathrm{~kJ}^{\mathrm{mol}}{ }^{-1}$ and $58.0 \pm 2.3 \mathrm{~kJ} . \mathrm{mol}$ 1 respectively (Table $1 \mathrm{~A} \& 1 \mathrm{~B}$ ). The corresponding experimental values ${ }^{46} 10.7 \pm 1.9 \mathrm{~kJ}^{\mathrm{mol}}{ }^{-1}$ and $55.0 \pm 3.4$ $\mathrm{kJ} \cdot \mathrm{mol}^{-1}$ are in agreement with the above results within the reported experimental error. The above experimental values and the reported evaluations correct the old literature values and reduce their uncertainties (old literature $\left(\Delta \mathrm{H}_{\mathrm{f}}{ }^{\circ}\right)_{\mathrm{m}}(\mathrm{g})$ values of $\mathrm{CH}_{2} \mathrm{ICl}: 13.6 \pm 20 \mathrm{~kJ} \cdot \mathrm{mol}^{-1}$ by Kudchadker et al. ${ }^{14}, 5 \pm 25 \mathrm{~kJ} \cdot \mathrm{mol}^{-1}$ by Skorobogatov et al. ${ }^{48}$ and of $\mathrm{CH}_{2} \mathrm{IBr}$ : $57.1 \pm 20 \mathrm{~kJ} \mathrm{~mol}^{-1}$ by Kudchadker et al. ${ }^{14}$ ).

The absolute errors with respect to experimental standard molar enthalpies of formation $\mid\left(\Delta \mathrm{H}_{\mathrm{f}}{ }^{\circ}\right)_{\mathrm{m}}(\mathrm{g})_{\text {experimental }}-\left(\Delta \mathrm{H}_{\mathrm{f}}{ }^{\circ}\right)_{\mathrm{m}}(\mathrm{g})_{\text {computed }}$ of molecules \#1\#16 (Table $1 \mathrm{~A} \& 1 \mathrm{~B}$, column EX-EV) were found to be lower than $2.4 \mathrm{~kJ} \cdot \mathrm{mol}^{-1}$ (exception $\mathrm{CHCl} 3$, absolute error: $4.9 \mathrm{~kJ} \cdot \mathrm{mol}^{-1}$ ). These values are below of the mean absolute deviation of $\sim 4.184 \mathrm{~kJ} \mathrm{~mol}^{-1}$ accuracy found for a large number of molecules with reliable experimental standard molar enthalpies of formation. The present results on halomethanes provide evidence that our approach is generally capable of predicting standard molar enthalpies of formation near to chemical accuracy.

Since these results support the reliability of our calculations the study was extended to the standard molar enthalpies of formation of mixed iodomethanes which are much less well established. Reliable experimental values could be found for only two compounds $\mathrm{CH}_{2} \mathrm{ICl}$ and $\mathrm{CH}_{2} \mathrm{IBr}$ described above - but several of them have been estimated by Gurvish et al. with uncertainties greater than $10 \mathrm{~kJ} \cdot \mathrm{mol}^{-1}$ (Table $1 \mathrm{~A} \& 1 \mathrm{~B})$.

The $\left(\Delta \mathrm{H}_{\mathrm{f}}{ }^{\circ}\right)_{\mathrm{m}}(\mathrm{g})$ average values $\left(\mathrm{kJ} . \mathrm{mol}^{-1}\right)$ of $\mathrm{CHI}_{2} \mathrm{Cl}$, $\mathrm{CHI}_{2} \mathrm{Br}, \mathrm{CHCl}_{2} \mathrm{I}$ and $\mathrm{CHBr}_{2} \mathrm{I}$ determined in this study $113.5 \pm 2.3(110.0 \pm 30), 165.4 \pm 4.7$ (165.0 \pm 35$)$, $7.0 \pm 2.3(5.0 \pm 20), 105.6 \pm 4.0$ (110.0 \pm 25$)$ respectively, are in agreement within the estimated uncertainty with the values reported in literature by Gurvish et al. ${ }^{15}$ (values in parentheses above and in Table $1 \mathrm{~A} \& 1 \mathrm{~B})$. They are also in agreement with the corresponding values $112.90,158.20,8.28$ and 93.51 $\mathrm{kJ} . \mathrm{mol}^{-1}$ reported by Kudchadker et $\mathrm{al}^{\mathbf{1 4}}$ with an uncertainty as high as $20.9 \mathrm{~kJ} \cdot \mathrm{mol}^{-1}$. However the large uncertainty in the literature values makes it difficult to draw any definitive conclusion regarding the accuracy of the method as it occurred for $\mathrm{CH}_{2} \mathrm{ICl}$ and $\mathrm{CH}_{2} \mathrm{IBr}$ where calculated values were within the 
reported narrow experimental errors. For these molecules additional theoretical studies are needed with high-accuracy calculations.

The present study was further extended to mixed halomethanes that are completely substituted with halogen atoms such as $\mathrm{CF}_{2} \mathrm{ClI}$, $\mathrm{CF}_{2} \mathrm{BrI}, \mathrm{CFCl}_{2} \mathrm{I}, \mathrm{CFI}_{2} \mathrm{Cl}$ and $\mathrm{CFBr}_{2} \mathrm{I}$. The reliable $\left(\Delta \mathrm{H}_{\mathrm{f}}{ }^{\circ}\right)_{\mathrm{m}}(\mathrm{g})$ average values $\left(\mathrm{kJ} \cdot \mathrm{mol}^{-1}\right)$ of the above compounds determined in this study $372.3 \pm 1.3(-380.0 \pm 25),-325.1 \pm 6.0 \quad(-$ $325.0 \pm 25),-176.3 \pm 3.3(-180.0 \pm 20),-60.5$ $\pm 7.4(-70 \pm 30)$ and $-72.3 \pm 7.2(-70 \pm 25)$ respectively are in agreement within the estimated uncertainty with the values reported in literature by Gurvish et al. ${ }^{15}$ (values in parentheses above and in Table 1A \& 1B). It is evident from the comparison of the calculated and experimental $\left(\Delta \mathrm{H}_{\mathrm{f}}{ }^{\circ}\right)_{\mathrm{m}}(\mathrm{g})$ values of the mixed halomethanes that the calculated value of $\mathrm{CF}_{2} \mathrm{BrI}-325.1 \pm 6.0 \mathrm{~kJ} \cdot \mathrm{mol}^{-1}$ coincides with the value reported by Gurvish et al. ${ }^{15}-325.0 \pm 25.0$ $\mathrm{kJ} . \mathrm{mol}^{-1}$ and is in good agreement with the value reported by Kudchadker et $\mathrm{al}^{14} 324.30 \mathrm{~kJ} \cdot \mathrm{mol}^{-1}$ (Table $1 \mathrm{~A} \& 1 \mathrm{~B}$ ). The calculated value of $\mathrm{CFCl}_{2} \mathrm{I}$ $176.3 \pm 3.3 \mathrm{~kJ} \mathrm{~mol}^{-1}$ is closer to the value reported by Kudchadker et al ${ }^{14} 178.2 \mathrm{~kJ} \cdot \mathrm{mol}^{-1}$ (Table 1A \& 1B) compared to the value reported by Gurvish et al. ${ }^{15}-180.0 \pm 20 \mathrm{~kJ} \cdot \mathrm{mol}^{-1}$. However, as mentioned above the large uncertainty in the literature values (20-30 $\mathrm{kJ} \cdot \mathrm{mol}^{-1}$ ) makes it difficult to draw any definitive conclusion regarding the accuracy of the method.
The absolute errors with respect to the experimental standard molar enthalpies of formation $\mid\left(\Delta \mathrm{H}_{\mathrm{f}}{ }^{\circ}\right)_{\mathrm{m}}(\mathrm{g})_{\text {experimental }}-\left(\Delta \mathrm{H}_{\mathrm{f}}{ }^{\circ}\right)_{\mathrm{m}}(\mathrm{g})_{\text {evaluated }}$ of the mixed halomethane molecules \#19-\#27 (Table $1 \mathrm{~A} \& \mathrm{~B}$, column EX-EV) were found to be lower than $4.5 \mathrm{~kJ} \cdot \mathrm{mol}^{-1}$ (exception $\mathrm{CF}_{2} \mathrm{ClI}$, absolute error: $7.7 \mathrm{~kJ} \mathrm{~mol}^{-1}$ and $\mathrm{CFI}_{2} \mathrm{Cl}$ absolute error: $9.5 \mathrm{~kJ}^{\mathrm{mol}}{ }^{-1}$ ) (Table 2).

The average of standard molar enthalpy of formation difference $\mid\left(\Delta H_{f}{ }^{0}\right)_{m}(g)_{\text {experimental }}$ $\left(\Delta \mathrm{H}_{\mathrm{f}}{ }^{\circ}\right)_{\mathrm{m}}(\mathrm{g})_{\text {evaluated }}$ of these compounds was equal to $3.7 \mathrm{~kJ} \cdot \mathrm{mol}^{-1}$ while the rms of $\left[\left(\Delta \mathrm{H}_{\mathrm{f}}{ }^{\circ}\right)_{\mathrm{m}}(\mathrm{g})_{\text {experimental }}-\left(\Delta \mathrm{H}_{\mathrm{f}}{ }^{\circ}\right)_{\mathrm{m}}(\mathrm{g})_{\text {evaluated }}\right]$ was found to be equal to $4.8 \mathrm{~kJ}^{\mathrm{mol}}{ }^{-1}$ that is well below the rms uncertainty of the experimental results (literature values) of $26.5 \mathrm{~kJ} \mathrm{~mol}^{-1}$ (Table 2).

The methodology described above that yielded comparably good agreement with experimental standard molar enthalpies of formation $\left(\Delta \mathrm{H}_{\mathrm{f}}{ }^{\circ}\right)_{\mathrm{m}}(\mathrm{g})$ was used to compute standard molar $\left(\Delta \mathrm{H}_{\mathrm{f}}{ }^{\circ}\right)_{\mathrm{m}}(\mathrm{g})$ of selected radicals and bond dissociation energies (BDE's) of the studied compounds in Table 1A \& 1B. These values were derived using reactions (R4 and R5) at the MP2/SPK-ATZP, DFT/BMK/SPK-ATZP and at the CCSD(T)(DLPNO)/SPK-ATZP level (Table 3). Optimization and frequency calculations were carried out at the DFT/BMK/SPK-ATZP and MP2/SPK-ATZP levels of theory. Single-point calculations at the $\operatorname{CCSD}(T) / S P K-A T Z P$ level were carried out on the MP2/SPK-ATZP optimized geometries.

$$
\begin{aligned}
& \mathrm{C}_{\mathrm{n}} \mathrm{H}_{2 \mathrm{n}+1} \mathrm{X} \longrightarrow \bullet \mathrm{C}_{\mathrm{n}} \mathrm{H}_{2 \mathrm{n}} \mathrm{X}+1 / 2 \mathrm{H}_{2} \\
& \mathrm{C}_{\mathrm{n}} \mathrm{H}_{2 \mathrm{n}} \mathrm{XY} \longrightarrow \cdot \mathrm{C}_{\mathrm{n}} \mathrm{H}_{2 \mathrm{n}-1} \mathrm{XY}+1 / 2 \mathrm{H}_{2}
\end{aligned}
$$

(where $X=F, C l, B r$, I and $n=1,2,3$. .) 
The bond dissociation energies (BDE's) of the studied compounds can be evaluated from calculated values of $\left(\Delta \mathrm{H}_{\mathrm{f}}{ }^{\circ}\right)_{\mathrm{m}}(\mathrm{g})$ for the neutral species (Table $1 \mathrm{~A} \& 1 \mathrm{~B}$ ) and their corresponding radicals (Table 3$)$ and the $\left(\Delta \mathrm{H}_{\mathrm{f}}{ }^{\circ}\right)_{\mathrm{m}}(\mathrm{g})$ values for chlorine $(121.301 \pm 0.008$ $\left.\mathrm{kJ} \cdot \mathrm{mol}^{-1}\right)^{10}$, bromine $\left(111.87 \pm 0.12 \mathrm{~kJ} \cdot \mathrm{mol}^{-1}\right)^{10}$ and iodine $\left(106.76 \pm 0.04 \mathrm{~kJ}^{\mathrm{mol}}{ }^{-1}\right)^{10}$

TABLE 2: Average value of the enthalpy difference $\left|\left(\Delta \mathrm{Hf}^{\circ}\right)_{\mathrm{m}}(\mathrm{g})\right|_{\text {diff }}\left(\mathrm{kJ} \cdot \mathrm{mol}^{-1}\right)$ between experimental (literature values) and evaluated data of selected mixed halomethanes in Table 1A \& 1B [where $\left|\left(\Delta \mathrm{Hf}^{\circ}\right)_{\mathrm{m}}(\mathrm{g})\right|_{\text {diff }}=\mid\left(\Delta \mathrm{Hf^{ \circ }}\right) \mathrm{m}(\mathrm{g})$ experimental $-\left(\Delta \mathrm{Hf}^{\circ}\right) \mathrm{m}(\mathrm{g})$ evaluated $\left.\mid\right]$.

\begin{tabular}{ccc}
$\begin{array}{c}\text { Compound \# } \\
\text { (Table 1A \& 1B) }\end{array}$ & Species & $\begin{array}{c}\left|\left(\Delta \mathrm{Hf}^{\circ}\right)_{\mathrm{m}}(\mathrm{g})\right|_{\text {diff }} \mathbf{a , b} \\
\left(\mathbf{k J . m o l} \mathbf{m}^{-\mathbf{1}}\right)\end{array}$ \\
\hline 19 & $\mathrm{CHI}_{2} \mathrm{Cl}$ & $3.5(30)$ \\
20 & $\mathrm{CHI}_{2} \mathrm{Br}$ & $0.4(35)$ \\
21 & $\mathrm{CHCl}_{2} \mathrm{I}$ & $2.0(20)$ \\
22 & $\mathrm{CHBr}_{2} \mathrm{I}$ & $4.4(25)$ \\
23 & $\mathrm{CF}_{2} \mathrm{ClI}$ & $7.7(25)$ \\
24 & $\mathrm{CF}_{2} \mathrm{BrI}$ & $0.1(25)$ \\
25 & $\mathrm{CFCl}_{2} \mathrm{I}$ & $3.7(20)$ \\
26 & $\mathrm{CFI}_{2} \mathrm{Cl}$ & $9.5(30)$ \\
27 & $\mathrm{CFBr}_{2} \mathrm{I}$ & $2.3(25)$ \\
Average & & $\mathbf{3 . 7}$ \\
rms & & $\mathbf{4 . 8 ( 2 6 . 5 )}$
\end{tabular}

a $\left(\Delta \mathrm{Hf}^{\circ}\right)_{\mathrm{m}}(\mathrm{g})$ at $298.15 \mathrm{~K}$ in units of $\mathrm{kJ} \cdot \mathrm{mol}^{-1}{ }^{\boldsymbol{b}}$ Values in parentheses represent reported experimental uncertainties of $\left(\Delta \mathrm{Hf}^{\circ}\right)_{\mathrm{m}}(\mathrm{g})$ (Table 1A \& 1B).

according to the definition of homolytic $X-Y$ bond cleavage enthalpy of reaction at 298.15 K (R6).

$$
\mathrm{R}-\mathrm{X} \rightarrow \cdot \mathrm{R}+\bullet \mathrm{X} \quad\left(\Delta \mathrm{H}_{\mathrm{r}}^{\circ}\right)_{\mathrm{m}}(\mathrm{g})=\mathrm{BDE}(\mathrm{R}-\mathrm{X})
$$

The standard molar enthalpies of formation $\left(\Delta \mathrm{H}_{\mathrm{f}}{ }^{\mathrm{O}}\right)_{\mathrm{m}}(\mathrm{g})$ of the radicals illustrated in Figure 4 were computed by the MP2/SPK-ATZP, DFT/BMK/SPK-ATZP and $\operatorname{CCSD}(T)(D L P N O) / S P K-A T Z P$ methods and show excellent correlation with the experimental values. The corresponding linear equations are (10), (11) and (12) with $R^{2}$ values $0.9990,0.9972$ and 0.9992 respectively.

$$
\begin{aligned}
& \left(\Delta \mathrm{H}_{\mathrm{f}}{ }^{\circ}\right)_{m(\text { (experimental) }}=1.0023 *\left(\Delta \mathrm{H}_{\mathrm{f}}{ }^{\circ}\right)_{\mathrm{m}(\mathrm{MP} 2 / \text { SPR-ATZP, reaction R5) }}-2.6720 ; \quad \mathrm{R}^{2}=0.9990 \\
& \left(\Delta \mathrm{H}_{\mathrm{f}}{ }^{\circ}\right)_{\mathrm{m}(\text { experimental })}=1.0365 *\left(\Delta \mathrm{H}_{\mathrm{f}}{ }^{\circ}\right)_{\mathrm{m}(\mathrm{DFT} / \mathrm{BMK} / \mathrm{SPK}-\mathrm{ATZP}, \text { reaction R5) }}+1.2666 ; \quad \mathrm{R}^{2}=0.9972 \\
& \left(\Delta \mathrm{H}_{\mathrm{f}}{ }^{\circ}\right)_{\mathrm{m}(\text { experimental })}=1.0184 *\left(\Delta \mathrm{H}_{\mathrm{f}}{ }^{\circ}\right)_{\mathrm{m}(\mathrm{CCSD}(\mathrm{T})(\mathrm{DLPNO}) / \text { SPR-ATZP,reactionR5) }}+2.1437 ; \mathrm{R}^{2}=0.9992
\end{aligned}
$$

The best correlations were obtained using the $\operatorname{CCSD}(\mathrm{T})(\mathrm{DLPNO}) / \mathrm{SPK}-\mathrm{ATZP}$ and MP2/SPK-ATZP methods. 
As shown in Table 3, the mean absolute error (MAE) between experimental and computed values $\mid\left(\Delta \mathrm{H}_{\mathrm{f}}{ }^{\circ}\right)_{\mathrm{m}}(\mathrm{g})_{\text {experimental }}-\left(\Delta \mathrm{H}_{\mathrm{f}}{ }^{\circ}\right)_{\mathrm{m}}(\mathrm{g})_{\text {computed }}$ of the organic radicals studied was found to be 3.1 and $4.6 \mathrm{~kJ} \cdot \mathrm{mol}^{-1}$ when the MP2/SPK-ATZP and CCSD(T)(DLPNO)/SPKATZP methods were used respectively. These values are close to the mean absolute deviation (MAE) of experimental standard molar enthalpies of formation of the same radicals that was calculated to be equal to $4.3 \mathrm{~kJ} \mathrm{~mol}^{-1}$. The DFT/BMK/SPK-ATZP method (MAE $=6.2$, Table 3 ) exhibited larger deviations from the experimental values.

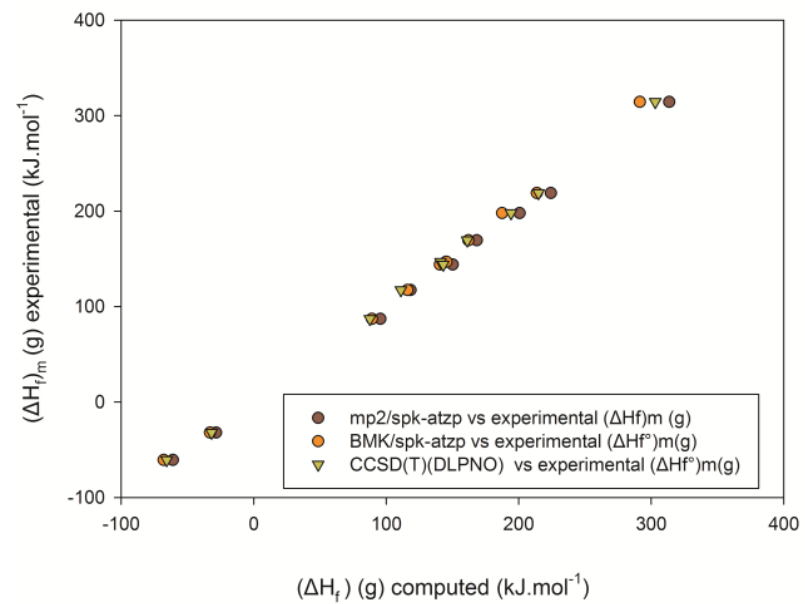

Figure 4. $\left(\Delta \mathrm{H}_{\mathrm{f}}\right)_{\mathrm{m}}(\mathrm{g})$ of radicals in Table 3 obtained at the MP2, BMK and CCSD(T)(DLPNO) level of theory and SPK-ATZP basis set using reactions R4 and R5 vs. their corresponding experimental values

The reliable $\left(\Delta \mathrm{H}_{\mathrm{f}}{ }^{\circ}\right)_{\mathrm{m}}(\mathrm{g})$ average values of the radicals listed in Table 3 were evaluated by extrapolating their computed values, which are obtained in this work, from correlation equations (10) - (12) and reactions R4 and R5. The reported uncertainties of evaluations correspond to estimated $95 \%$ confidence limits.

The reliable $\left(\Delta \mathrm{H}_{\mathrm{f}}{ }^{\circ}\right)_{\mathrm{m}}(\mathrm{g})$ average value of the $\mathrm{CH}_{3}$ radical was found to be $146.8 \pm 0.3$ $\mathrm{kJ} \cdot \mathrm{mol}^{-1}$ in agreement within the estimated uncertainty with the experimental value reported by $\mathrm{Luo}^{13} 146.7 \pm 0.3 \mathrm{~kJ}^{13} \mathrm{~mol}^{-1}$ and within twice the experimental uncertainty reported by Ruscic et al. ${ }^{60} 145.7 \pm 0.8 \mathrm{~kJ} \cdot \mathrm{mol}^{-1}$ (Table 3). The reliable $\left(\Delta \mathrm{H}_{\mathrm{f}}{ }^{\circ}\right)_{\mathrm{m}}(\mathrm{g})$ average value of the $\mathrm{CH}_{2} \mathrm{~F}$ radical $-31.5 \pm 3.3 \mathrm{~kJ} \cdot \mathrm{mol}^{-1}$ is also in good agreement -within the estimated uncertainty- with the values reported by Pickard et al ${ }^{61}$ and McMillen et al. ${ }^{62}-32 \pm 8$ and $-32.6 \pm 8.4 \mathrm{~kJ} \cdot \mathrm{mol}^{-1}$ respectively. Similarly, the computed $\left(\Delta \mathrm{H}_{\mathrm{f}}{ }^{\circ}\right)_{\mathrm{m}}(\mathrm{g})$ average values of $\mathrm{CH}_{2} \mathrm{Cl} 115.5 \pm 4.5 \mathrm{~kJ}^{-\mathrm{mol}^{-1}}, \mathrm{CHBr}_{2} 198.0 \pm 5.5 \mathrm{~kJ} \cdot \mathrm{mol}^{-1}, \mathrm{CHClF}-65.9 \pm$ $7.0 \mathrm{~kJ} \cdot \mathrm{mol}^{-1}$ and $\mathrm{CHI}_{2} 311.2 \pm 5.8 \mathrm{~kJ} \cdot \mathrm{mol}^{-1}$ agree well within the experimental uncertainty with the values reported by $\mathrm{Luo}^{13} 117.3 \pm 3.1 \mathrm{~kJ}^{13} \mathrm{~mol}^{-1}$, Davalos et al. ${ }^{47}$ $197.8 \pm 4.3 \mathrm{~kJ} \cdot \mathrm{mol}^{-1}$, Seetula et al. ${ }^{64}-60.7 \pm 10 \mathrm{~kJ} \cdot \mathrm{mol}^{-1}$ and Seetula et al. ${ }^{68} 314.4 \pm$ $3.3 \mathrm{~kJ} \cdot \mathrm{mol}^{-1}$ respectively (Table 3 ). 
The reliable $\left(\Delta \mathrm{H}_{\mathrm{f}}{ }^{\circ}\right)_{\mathrm{m}}(\mathrm{g})$ average values of $\mathrm{CH}_{2} \mathrm{Br}$ radical $166.2 \pm 1.3 \mathrm{~kJ} \cdot \mathrm{mol}^{-1}, \mathrm{CH}_{2} \mathrm{I} 222.4$ $\pm 4.0 \mathrm{~kJ} \cdot \mathrm{mol}^{-1}$ and $\mathrm{CHBrCl} 147.8 \pm 0.6 \mathrm{~kJ} \mathrm{~mol}^{-1}$ were also evaluated and found to be within twice the experimental uncertainty reported by Davalos et al. ${ }^{47} 169.4 \pm 3.0$ $\mathrm{kJ} \cdot \mathrm{mol}^{-1}, 215.7 \pm 6.7 \mathrm{~kJ} \cdot \mathrm{mol}^{-1}$ and $143.9 \pm 3.7 \mathrm{~kJ} \mathrm{~mol}^{-1}$ respectively (Table 3).

As shown in Table 3, the MAE and rms errors of the radical evaluations were found to be equal to $3.6 \mathrm{~kJ} \cdot \mathrm{mol}^{-1}$ and $4.1 \mathrm{~kJ} \cdot \mathrm{mol}^{-1}$ respectively. These values are below the corresponding MAE and rms uncertainty of the experimental results (literature values) of 4.3 and $5.2 \mathrm{~kJ}^{\mathrm{mol}}{ }^{-1}$ (Table 3).

Since these results support the reliability of our calculations the study was extended to the standard molar enthalpies of formation of $\mathrm{CHICl}$ and $\mathrm{CHIBr}$. Experimental standard molar enthalpies of formation of these radicals are not available in the literature to the best of our knowledge. The reliable $\left(\Delta \mathrm{H}_{\mathrm{f}}{ }^{\circ}\right)_{\mathrm{m}}(\mathrm{g})$ average values of $\mathrm{CHICl}$ and $\mathrm{CHIBr}$ radicals computed here using the method described above were found to be $200.1 \pm$ $4.5 \mathrm{~kJ} \cdot \mathrm{mol}^{-1}$ and $249.7 \pm 3.3 \mathrm{~kJ} \cdot \mathrm{mol}^{-1}$ respectively.

The bond dissociation energies (BDE's) of the studied compounds were subsequently derived from reaction R6 using the most reliable evaluations of $\left(\Delta \mathrm{H}_{\mathrm{f}}{ }^{\circ}\right)_{\mathrm{m}}(\mathrm{g})$ for the neutral species (Table $1 \mathrm{~A} \& 1 \mathrm{~B}$ ) and their corresponding radicals (Table 3 ) and the $\left(\Delta \mathrm{H}_{\mathrm{f}}{ }^{\circ}\right)_{\mathrm{m}}(\mathrm{g})$ values for fluorine $(79.36 \pm 0.048)$, chlorine $\left(121.228 \pm 0.0011 \mathrm{~kJ} \cdot \mathrm{mol}^{-1}\right)^{\mathbf{4 3}}$, bromine $\left(111.855 \pm 0.056 \mathrm{~kJ}^{\mathrm{mol}}{ }^{-1}\right)^{43}$ and iodine $\left(106.757 \pm 0.0021 \mathrm{~kJ} \cdot \mathrm{mol}^{-1}\right)^{43}$. These values are shown in Table 4 where the corresponding BDE's calculated at the MP2/SPKATZP and CCSD(T)/SPK-ATZP level are also listed.

The rms error of the evaluated BDE values was found to be equal to 4.9 compared to $8.2 \mathrm{~kJ} \mathrm{~mol}^{-1}$ of the experimental/literature values (Table 4).

For most of the above BDE evaluations good agreement was observed between calculated and experimental/literature values (deviations less than $4.8 \mathrm{~kJ} \cdot \mathrm{mol}^{-1}$ ). For a few of the iodinated-molecules and for a fluorine containing molecule $\left(\mathrm{Cl}_{-}-\mathrm{CH}_{2} \mathrm{I}, \mathrm{I}-\mathrm{CH}_{2} \mathrm{Br}\right.$, $\mathrm{F}-\mathrm{CH}_{2} \mathrm{Cl}$ ) deviations were found to be around $6.0 \mathrm{~kJ} \cdot \mathrm{mol}^{-1}$. The largest difference was observed for $\mathrm{Br}_{-} \mathrm{CHBr}_{2}\left(12.4 \mathrm{~kJ} \mathrm{~mol}^{-1}\right.$, in agreement with the difference reported by S.W. North et al. ${ }^{70} 12.6 \mathrm{~kJ} \cdot \mathrm{mol}^{-1}$ at the MP2 and $\mathrm{CCSD}(\mathrm{T})$ levels of theory), $\mathrm{Cl}-\mathrm{CHClBr}(-9.2$ $\mathrm{kJ} \cdot \mathrm{mol}^{-1}$ ) and $\mathrm{Cl}-\mathrm{CHFCl}\left(7.9 \mathrm{~kJ} \cdot \mathrm{mol}^{-1}\right)$. However, the BDE values reported for the above species in this paper are within experimental error with the literature values where large associated uncertainties were observed $13.0,17.0$ and $13.4 \mathrm{~kJ}^{\mathrm{mol}}{ }^{-1}$ respectively. The estimated BDE values reported for $\mathrm{Br}^{-} \mathrm{CHBr}_{2}$ and $\mathrm{Cl}-\mathrm{CHClBr}$ by Davalos et al ${ }^{47} 254.3 \pm$ $5.4 \mathrm{~kJ} \cdot \mathrm{mol}^{-1}$ and $316.1 \pm 4.4 \mathrm{~kJ} \cdot \mathrm{mol}^{-1}$ respectively are in better agreement to the ones reported in this work (Table 4). Only for five of the iodinated-molecules in Table 4 experimental/literature $\operatorname{BDE}(\mathrm{C}-\mathrm{X})$ values are available to the best of our knowledge. The $\mathrm{BDE}(\mathrm{C}-\mathrm{X})$ values reported in this study for $\mathrm{Cl}_{-} \mathrm{CH}_{2} \mathrm{I}, \mathrm{Br}-\mathrm{CH}_{2} \mathrm{I}, \mathrm{I}-\mathrm{CH} 2 \mathrm{Cl}$ and I-CH2I are in agreement within the estimated uncertainty with the experimental values reported in literature. The BDE value of I-CH2Br evaluated and reported in Table 4 was found to be 
within twice the experimental uncertainty of the value estimated and reported by Davalos et al. $^{47}$ but within the experimental uncertainty of the value determined by Lago $^{46}$. For the rest of the iodinated-molecules reliable BDE values are reported with estimated $95 \%$ confidence limits ranging from 3.7 to $7.5 \mathrm{~kJ} \cdot \mathrm{mol}^{-1}$.

Correlation equations of experimental/literature vs. calculated BDE values were also obtained (13-15) where the best correlations were observed for the CCSD(T)/SPK-ATZP method and for the evaluated BDE's (Table 4):

$$
\begin{aligned}
& \mathrm{BDE}(\mathrm{C}-\mathrm{X})_{(\text {experimental) }}=1.0179 * \mathrm{BDE}(\mathrm{C}-\mathrm{X})_{\text {MP2/SPK-ATZP_Table 4 }}-7.1392 ; \mathrm{R}^{2}=0.9956 \\
& \mathrm{BDE}(\mathrm{C}-\mathrm{X})_{(\text {experimental) }}=0.9954 * \mathrm{BDE}(\mathrm{C}-\mathrm{X})_{\mathrm{CCSD}(\mathrm{T} / \text { /SPK-ATZP_Table } 4}+6.8619 ; \mathrm{R}^{2}=0.9965 \\
& \mathrm{BDE}(\mathrm{C}-\mathrm{X})_{(\text {experimental) }}=1.0083 * \mathrm{BDE}(\mathrm{C}-\mathrm{X})_{\text {evaluations_Table } 4}-2.0992 ; \mathrm{R}^{2}=0.9965
\end{aligned}
$$

These correlation equations include the iodinated molecules of Table 4 with known experimental BDE values. The BDE values of the molecules of Table 4 - illustrated in Figure 5 - show excellent correlation with the experimental values. The corresponding linear equations (13), (14) and (15) have $R^{2}$ values 0.9956, 0.9965 and 0.9965 respectively.

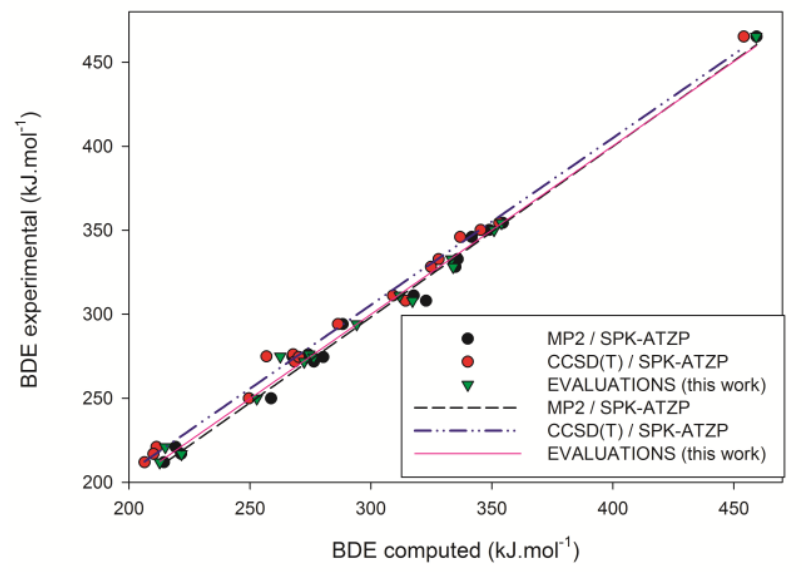

Figure 5. Carbon-halogen $\mathrm{BDE}(\mathrm{C}-\mathrm{X}, \mathrm{X}=\mathrm{F}, \mathrm{Cl} \mathrm{Br}, \mathrm{I})$ of the molecules in Table 4 obtained at MP2/SPK-ATZP \& CCSD(T)/SPKATZP levels of theory and by evaluations (this paper) vs. their corresponding experimental values $\left(\mathrm{kJ} \cdot \mathrm{mol}^{-1}\right)$.

On the basis of these results, the method described above, together with isodesmic reactions to remove errors, may profitably be used to obtain very accurate standard molar enthalpies of formation of halomethane species. 


\section{CONCLUSIONS}

In this study standard molar enthalpies of formation $\left(\Delta \mathrm{H}_{\mathrm{f}}{ }^{\circ}\right)_{\mathrm{m}}(\mathrm{g})$ and carbon-halogen BDE of a set of halogen-containing organic species with atmospheric importance have been calculated. Ab initio calculations were performed at the DFT, MP2 and CCSD(T) levels of theory via isodesmic reaction schemes which can provide very high systematic error compensation. The obtained results were compared to theoretical as well as experimental data available in the literature and thermochemical databases. In all cases, excellent correlations were observed and reliable $\left(\Delta \mathrm{H}_{\mathrm{f}}{ }^{\circ}\right)_{\mathrm{m}}(\mathrm{g})$ average values of the species studied were obtained by extrapolating their theoretical values from the corresponding correlation equations and isodesmic reactions.

The application of isodesmic reactions to remove the systematic errors yields $\left(\Delta \mathrm{H}_{\mathrm{f}}{ }^{\circ}\right)_{\mathrm{m}}(\mathrm{g})$ that are in close agreement with experimental values, with rms deviations that range from $2.8 \mathrm{~kJ}^{-\mathrm{mol}^{-1}}[\mathrm{CCSD}(\mathrm{T})]$ to $5.1 \mathrm{~kJ} \cdot \mathrm{mol}^{-1}$ [MP2] which is well below the rms experimental uncertainty of $16.1 \mathrm{~kJ} \cdot \mathrm{mol}^{-1}$. Similarly, the calculated $\left(\Delta \mathrm{H}_{\mathrm{f}}{ }^{\circ}\right)_{\mathrm{m}}(\mathrm{g})$ values for the corresponding halomethane radicals are found to be in close agreement with experimental values, with rms deviations that range from $4.1 \mathrm{~kJ} . \mathrm{mol}^{-1}$ [MP2] to 5.7

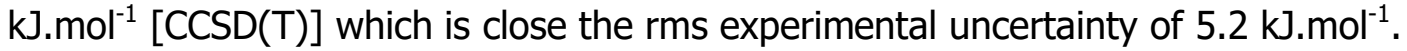

The reliable average carbon-halogen BDE values are also in close agreement with experimental values with rms deviation $4.9 \mathrm{~kJ} \cdot \mathrm{mol}^{-1}$ which is well below the rms experimental uncertainty of $8.2 \mathrm{~kJ} \cdot \mathrm{mol}^{-1}$.

Based on this approach, new estimates for standard molar enthalpies of formation and $\mathrm{BDE}$ are suggested including for species that to our knowledge there are no experimental $\left(\Delta \mathrm{H}_{\mathrm{f}}{ }^{\circ}\right)_{\mathrm{m}}(\mathrm{g})\left(\mathrm{CHCl}_{2} \mathrm{Br}, \mathrm{CHBr}_{2} \mathrm{Cl}, \mathrm{CHBrCl}, \mathrm{CHICl}, \mathrm{CHIBr}\right)$ or $\mathrm{BDE}$ values $\left(\mathrm{CHCl}_{2} \mathrm{Br}, \mathrm{CHBr}_{2} \mathrm{Cl}, \mathrm{CHBrCl}, \mathrm{CHICl}, \mathrm{CHIBr}\right)$ available in the literature. Reliable $\left(\Delta \mathrm{H}_{\mathrm{f}}{ }^{\circ}\right)_{\mathrm{m}}(\mathrm{g})$ and $\mathrm{BDE}$ average values are also suggested for several halomethanes where literature experimental or evaluation data have large error bars ( $>10 \mathrm{~kJ})$.

On the basis of these results, it is concluded that these calculational procedures at the MP2 and particularly at the $\operatorname{CCSD}(\mathrm{T})$ level, together with isodesmic reactions and correlation and regression analysis may be used to obtain accurate standard molar enthalpies of formation $\left(\Delta \mathrm{H}_{\mathrm{f}}{ }^{\circ}\right)_{\mathrm{m}}(\mathrm{g})$ and carbon-halogen BDE of halomethane species. 


\section{DISCLOSURE STATEMENT}

No potential conflict of interest is reported by the author.

\section{ORCID}

Konstantinos Kalamatianos

https://orcid.org/0000-0002-0276-6531

\section{CONTACT}

K.G. Kalamatianos kgkalamatianos@gmail.com Physical and Computational Chemistrygy Laboratory, I.Y.A., Athens, Greece

\section{REFERENCES}

(1) Bomble, Y.J.; Vazquez, J; Kallay, M., Michauk, C; Szalay, P.G.; Csaszar, A.G.; Gauss, J.; Stanton, J.F. High-accuracy extrapolated ab initio thermochemistry. II. Minor improvements to the protocol and a vital simplification. J. Chem. Phys. 2006, 125, 064108/1- 064108/8.

(2) Harding, M.E.; Vazquez, J; Ruscic, B.; Wilson, A.K.; Gauss, J.; Stanton, J.F. Highaccuracy extrapolated ab initio thermochemistry. III. Additional improvements and overview. J. Chem. Phys. 2008, 128, 11, 114111/1 - 114111/15

(3) Martin, J.M.L.; de Oliveira, G. Towards standard methods for benchmark quality ab initio thermochemistry-W1 and W2 theory. J. Chem. Phys. 1999, 111, 18431856.

(4) Kanton, A.; Rabinovich, E; Martin, J.M.L.; Ruscic, B. W4 theory for computational thermochemistry: In pursuit of confident sub- $\mathrm{kJ} / \mathrm{mol}$ predictions. J. Chem. Phys. 2006, 125, 144108/1-144108/17.

(5) Irikura, K.K.; Frurip, D.J.; Computational Thermochemistry, ACS Symposium Series 667; American Chemical Society, 1998

(6) Fabian, W.M.F.; Accurate thermochemistry from quantum chemical calculations? Monatsh. Chem. 2008, 139, 309-318.

(7) Manzetti, S.; van der Spoel, E.R.; van der Spoel, D. Chemical properties, environmental fate, and degradation of seven classes of pollutants Chem. Res. Toxicol. 2014, 27, 713-717.

(8) Walsh, A. Inorganic materials: The quest for new functionality. Nat. Chem. 2015, 7, 274-275. 
(9) Ruscic, B.; Pinzon, R.; Laszewski, von G.; Kodeboyina, D.; Burcat, A.; Leahy, D.; Montoya, D.; Wagner, A.F. Active Thermochemical Tables: thermochemistry for the $21^{\text {st }}$ century. Journal of Physics: Conference Series 2005, 16, 561-570.

(10) NIST Chemistry Webbook in: Linstrom P.J.; Mallard W.G. (Eds.), NIST Standard Reference Database Number 69, National Institute of Standards and Technology: Gaithersburg, MD, 20899.

(11) Pedley, J.B. Thermochemical Data and Structures of Organic Compounds, 1994, 1 , Thermodynamics Research Center, College Station, TX.

(12) Burkholder, J.B.; Sander, S.P.; Abbatt, J.P.D.; Barker, J.R.; Huie, R.E.; Colb, C.E.; Kurylo, M.J.; Orkin, V.L.; Wilmouth, D.M.; Wine, P.H. Chemical kinetics and photochemical data for use in atmospheric studies. Evaluation No. 18, 2015, NASA jet Propulsion Laboratory Publication, California Institute of Technology, CA.

(13) Luo, Y.-R. Comprehensive Handbook of Chemical Bond Energies, 2007, CRC Press, Boca Raton, FL.

(14) Kudchadker, S.A.; Kudchadker, A.P. Ideal Gas Thermodynamic Properties of CH4-

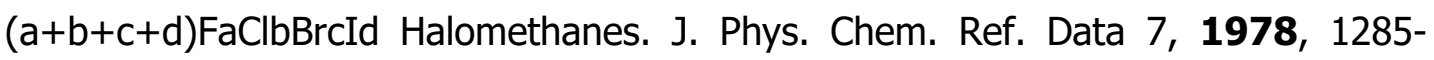
1307.

(15) Gurvich, L.V.; Veyts, I.V.; Alcock C.B. (Eds.) Thermodynamic Properties of Individual Substances. 4th ed.; Hemisphere, New York 1991; Vol. 2.

(16) Class,T.; Ballschmiter, K. J. Chemistry of organic traces in air: Sources and distribution of bromo- and bromochloromethanes in marine air and surfacewater of the Atlantic Ocean. Atmos. Chem. 1988, 6, 35-46.

(17) Alicke, B.; Hebestreit, K.; Stutz, J.; Platt, U. Iodine oxide in the marine boundary layer. Nature, 1999, 397, 572-573.

(18) Heumann, K.G. Determination of inorganic and organic traces in the clean room compartment of Antarctica. Anal. Chim. Acta, 1993, 283, 230-245.

(19) Mossigner, J.C.; Shallcross, D.E.; Cox, R.A. UV-VIS absorption cross-sections and atmospheric lifetimes of $\mathrm{CH} 2 \mathrm{Br} 2, \mathrm{CH} 2 \mathrm{I} 2$ and $\mathrm{CH} 2 \mathrm{BrI}$. J. Chem. Soc., Faraday Trans., 1998, 94, 1391-1396.

(20) Pople, J.A.; Head-Gordon, M.; Fox, D.J; Raghavachari, K.; Curtiss, L.A. Gaussian-1 theory: A general procedure for prediction of molecular energies. J. Chem. Phys., 1989, 90, 5622-5629.

(21) Curtiss, L.A.; Jones, C.; Trucks, G.W.; Raghavachari, K.; Pople, J.A. Gaussian-1 theory of molecular energies for second-row compounds. J. Chem. Phys., 1990, 93, 2537-2545.

(22) Curtiss, L.A.; Raghavachari, K.; Trucks, G.W.; Pople, J.A. Gaussian-2 theory for molecular energies of first- and second-row compounds. J. Chem. Phys., 1991, 94, 7221-7230.

(23) Martin, J.M.L.; de Oliveira, G. Towards standard methods for benchmark quality ab initio thermochemistry-W1 and W2 theory. J. Chem. Phys., 1999, 111, 18431856. 
(24) Parthiban, S.; Martin, J.M.L. Assessment of $W 1$ and $W 2$ theories for the computation of electron affinities, ionization potentials, heats of formation, and proton affinities. J. Chem. Phys., 2001, 114, 6014-6029.

(25) Simmie, J.M.; Somers, K.P. Benchmarking Compound Methods (CBS-QB3, CBSAPNO, G3, G4, W1BD) against the Active Thermochemical Tables: A Litmus Test for Cost-Effective Molecular Formation Enthalpies. J. Phys. Chem. A, 2015, 119, 7235-7246.

(26) World Meteorological Organization (WMO), Global Ozone Research and Monitoring Project - Report No 56, 2014, Geneva, Switzerland

(27) Coffin, C. J.; Ge, R.; Yang, S.; Kramer, P.M.; Tao, L.; Pereira, M.A. Effect of Trihalomethanes on Cell Proliferation and DNA Methylation in Female B6C3F1 Mouse Liver. Toxicological Sciences, 2000, 58, 243-252.

(28) Granovsky, A.A. Firefly version 8.2.0 http://classic.chem.msu.su/gran/firefly/index.html

(29) Schmidt, M.W.; Baldridge, K.K.; Boatz, J.A.; Elbert, S.T.; Gordon, M.S.; Jensen, J.H.; Koseki, S.; Matsunaga, N.; Nguyen, K.A.; Su, S.; Windus, T.L.; Dupuis, M.; Montgomery, J.A. General atomic and molecular electronic structure system. J. Comput. Chem., 1993, 14, 1347-1363.

(30) Gordon, M.S.; Schmidt, M.W. Advances in electronic structure theory: GAMESS a decade later in Theory and Applications of Computational Chemistry: the first forty years Dykstra, C.E.; Frenking, G.; Kim, K.S.; Scuseria, G.E. (editors), Elsevier, Amsterdam, 2005, 1167-1189.

(31) Neese, F. The Orca program system. Comput. Mol. Sci., 2012, 2, Issue 1, 73-78.

(32) Neto, A.C.; Muniz, E.P.; Centoducatte, R.; Jorge, F.E. Gaussian basis sets for correlated wave functions. Hydrogen, helium, first- and second-row atoms. J. Mol. Struct. Theochem, 2005, 718, 219-224.

(33) Camiletti, G.G.; Neto, A.C.; Jorge, F.E.; Machado, S.F. Augmented Gaussian basis sets of double and triple zeta valence qualities for the atoms $\mathrm{K}$ and $\mathrm{Sc}-\mathrm{Kr}$ : Applications in HF, MP2, and DFT calculations of molecular electric properties. J. Mol. Struct. Theochem, 2009, 910, 122-125.

(34) Becke, A.D. Density-functional thermochemistry. III. The role of exact exchange J. Chem. Phys., 1993, 98, 5648-5652.

(35) Raghavachari, K.; Trucks, G.W.; Pople, J.A.; Head-Gordon, M. A fifth-order perturbation comparison of electron correlation theories. Chem. Phys. Lett., 1989, 157, 479-480.

(36) Minenkov, Y.; Bistoni, G.; Riplinger, C.; Auer, A.A.; Neese, F.; Cavallo, L. Pair natural orbital and canonical coupled cluster reaction enthalpies involving light to heavy alkali and alkaline earth metals: the importance of sub-valence correlation, Physical Chemistry Chemical Physics, 2017, 19, 9374-9391.

(37) Paulechka, E.; Kazakov, A.; Efficient DLPNO-CCSD(T)-based estimation of formation enthalpies for $\mathrm{C}_{-}, \mathrm{H}-, \mathrm{O}-$, and $\mathrm{N}$-containing closed-shell compounds validated against critically evaluated experimental data, J. Phys. Chem. A, 2017, 121, 4379-4387. 
(38) Noro, T.; Sekiya, M.; Koga, T. Correlating basis sets for the $\mathrm{H}$ atom and the alkalimetal atoms from Li to Rb. Theor. Chem. Acc., 2003, 109, 85-90.

(39) Noro, T.; Sekiya, M.; Koga, T. Contracted polarization functions for the atoms helium through neon. Theor. Chem. Acc., 1997, 98, 25-32.

(40) Hehre, W.J.; Ditchfield, R., Radom, I.; Pople, J.A. Molecular orbital theory of the electronic structure of organic compounds. V. Molecular theory of bond separation. J. Am. Chem. Soc., 1970, 92, 4796-4801.

(41) Glukhovtsev, M.N.; Laiter, S. High levelab initio stabilization energies of benzene. Theor. Chim. Acta, 1995, 92, 327-332.

(42) Nicolaides, A.; Radom, I. An evaluation of the performance of G2, G2(MP2) and G2(MP2,SVP) theories for heats of formation and heats of reaction in the case of 'large' hydrocarbons. Mol. Phys., 1996, 88, 759-766.

(43) Argonne National Laboratory, Active Thermochemical Tables, ATcT https://atct.anl.gov/

(44) Shuman, N.S.; Zhao, L.Y.; Boles, M.; Baer, T.; Sztaray, B., J. Phys. Chem. A, 2008, 112, 10533-10534.

(45) Papina, T.S.; Kolesov, V.P.; Golovanova, Y.G., The standard enthalpy of formation of bromoform. Russ. J. Phys. Chem. (Engl. Transl.), 1982, 56, 16661668.

(46) Lago, A.F.; Kercher, J.P.; Bodi, A.; Sztaray, B.; Miller, B.; Wurzelmann, D.; Baer, T. Dissociative Photoionization and Thermochemistry of Dihalomethane Compounds Studied by Threshold Photoelectron Photoion Coincidence Spectroscopy. J. Phys. Chem. A, 2005, 109, 1802-1809.

(47) Davalos, J.Z.; Notario, R.; Cuevas, C.A.; Oliva, J.M.; Saint-Lopez, A., Thermochemistry of halogen-containing organic compounds with influence on atmospheric chemistry. Computational and Theoretical Chemistry, 2017, 1099, 36-44.

(48) Skorobogatov, G.A. ; Dymov, B.P.; Nedozrelova, Equilibrium and rate constants of the reactions $\mathrm{RI} \leftrightarrows \mathrm{R}+\mathrm{I}$ and $\mathrm{I}+\mathrm{RI} \leftrightarrows \mathrm{I}_{2}+\mathrm{R}\left(\mathrm{R}=\mathrm{CH}_{2} \mathrm{Cl}, \mathrm{CH}_{2} \mathrm{I}\right)$ at $300-700^{\circ} \mathrm{C}$. I.V. Russ. J. Gen. Chem (Engl. Transl.), 1996, 66, 1786-1792.

(49) Baer, T.; Walker, S.H.; Shuman, N.S.; Bodi, A. One- and Two-Dimensional Translational Energy Distributions in the Iodine-Loss Dissociation of 1,2$\mathrm{C}_{2} \mathrm{H}_{4} \mathrm{I}_{2}{ }^{+}$and $1,3-\mathrm{C}_{3} \mathrm{H}_{6} \mathrm{I}_{2}{ }^{+}$: What Does This Mean? J. Phys. Chem. A, 2012, 116, 2833-2844.

(50) Cox, J.D.; Pilcher, G. "Thermochemistry of Organic and Organometallic Compounds" Academic, London, 1970.

(51) Manion, J.A. Evaluated enthalpies of formation of the stable closed shell $\mathrm{C} 1$ and C2 chlorinated hydrocarbons. J. Phys. Chem. Ref. Data, 2002, 31, 124-165.

(52) Bodi, A.; Shuman, N.S.; Baer, T. On the ionization and dissociative photoionization of iodomethane: a definitive experimental enthalpy of formation of CH3I. Phys. Chem. Chem. Phys., 2009, 11, 11013-11021. 
(53) Chase, M.W. Jr., NIST-JANAF Thermochemical Tables, 4th Edition, J. Phys. Chem. Ref. Data, Monograph 9, 1998, 1-1951.

(54) Berry, R. J.; Burgess, D.R.F, Jr.; Nyden, M.R.; Zachariah, M.R; Melius, C.F.; Schwartz, M., Halon Thermochemistry: Calculated Enthalpies of Formation of Chlorofluoromethanes. J. Phys. Chem, 1996, 100, 7405-7410.

(55) Yaws, C.L. Yaw's Handbook of Thermodynamic Properties for Hydrocarbons and Chemicals, Knovel, 2009.

(56) Baibuz, V.F., Explosion method and heat of formation of CF4, CFCl, CFCl3, and CCl4. Dokl. Phys. Chem. (Engl. Transl.), 1961, 140, 786-788.

(57) Bodi, A.; Kvaran, A.; Sztaray, B., Thermochemistry of Halomethanes $\mathrm{CF}_{\mathrm{n}} \mathrm{Br}_{4}-$ $\mathrm{n}(\mathrm{n}=0-3)$ Based on iPEPICO Experiments and Quantum Chem. Computations. J. Phys. Chem. A, 2011, 115, 13443-13451.

(58) Lide, D.R. CRC Handbook of Chemistry and Physics, 90th Edition, CRC Press: Cleveland, Ohio, 2009.

(59) Harvey, J.; Tuckett, R.P.; Bodi, A., A Halomethane Thermochemical Network from iPEPICO Experiments and Quantum Chemical Calculations. J. Phys. Chem. A, 2012, 116, 9696-9705.

(60) Ruscic, B.; Boggs, J.E.; Burcat, A.; Gsaszar, A.G.; Demaison, J.; Janoscheck, R.; Martin, J.M.L.; Morton, M.L.; Rossi, M.J; Stanton, J.F.; Szalay, P.G.; Westmoreland, P.R.; Zabel, F.; Berces, T.; J. Phys. Chem. Ref. Data, 2005, 34, 573-656.

(61) Pickard, J.M.; Rodgers, A. S. Kinetics of the gas-phase reaction $\mathrm{CH} 3 \mathrm{~F}+\mathrm{I} 2 \leftrightarrows$ $\mathrm{CH} 2 \mathrm{FI}+\mathrm{HI}$ : The $\mathrm{C} \cdot \mathrm{H}$ bond dissociation energy in methyl and methylene fluorides Int. J. Chem. Kinet. 1983, 15, 569-577.

(62)McMillen, D. F.; Golden, D. M., Hydrocarbon Bond Dissociation Energies. Ann. ReV. Phys. Chem. 1982, 33, 493-532.

(63) Seetula, J.A., Kinetics of the $\mathrm{R}+\mathrm{HBr} \rightleftarrows \mathrm{RH}+\mathrm{Br}\left(\mathrm{R}=\mathrm{CH}_{2} \mathrm{Br}\right.$, $\mathrm{CHBrCl}$ or $\left.\mathrm{CCl}_{3}\right)$ equilibrium. Thermochemistry of the $\mathrm{CH}_{2} \mathrm{Br}$ and $\mathrm{CHBrCl}$ radicals. Phys. Chem. Chem. Phys., 2003, 5, 849-855.

(64) Tschuikow-Roux, $\mathrm{E}$.; Paddison, $\mathrm{S}$. Bond dissociation energies and radical heats of formation in $\mathrm{CH}_{3} \mathrm{Cl}, \mathrm{CH}_{2} \mathrm{Cl}_{2}, \mathrm{CH}_{3} \mathrm{Br}, \mathrm{CH}_{2} \mathrm{Br}_{2}, \mathrm{CH}_{2} \mathrm{FCl}$, and $\mathrm{CHFCl}_{2}$. Int. J. Chem. Kinet., 1987, 19, 15-24.

(65) Seetula, J.A; Eskola, A., Kinetics of the $\mathrm{R}+\mathrm{HBr} \rightleftarrows \mathrm{RH}+\mathrm{Br}\left(\mathrm{CH}_{3} \mathrm{CHBr}, \mathrm{CHBr}_{2}\right.$ or $\mathrm{CDBr}_{2}$ ) equilibrium: Thermochemistry of the $\mathrm{CH}_{3} \mathrm{CHBr}$ and $\mathrm{CHBr}_{2}$ radicals. J. Chem. Phys., 2008, 351, 141-146.

(66) Lago, A.F.; Baer T., Dissociation dynamics and thermochemistry of chloroform and tetrachloroethane molecules studied by threshold photoelectron photoion coincidence. Int. J. Mass. Spectrom. 2006, 252, 20-25.

(67) Seetula, J.A. Chem. Soc. Faraday Trans., Kinetics and thermochemistry of the $\mathrm{R}+$ $\mathrm{HBr} \rightleftharpoons \mathrm{RH}+\mathrm{Br}\left(\mathrm{R}=\mathrm{CH}_{2} \mathrm{Cl}, \mathrm{CHCl}_{2}, \mathrm{CH}_{3} \mathrm{CHCl}\right.$ or $\left.\mathrm{CH}_{3} \mathrm{CCl}_{2}\right)$ equilibrium. 1996, 92, 3069-3078. 
(68) Seetula, J.A., Kinetics of the $\mathrm{R}+\mathrm{HBr} \rightarrow \mathrm{RH}+\mathrm{Br}\left(\mathrm{R}=\mathrm{CH}_{2} \mathrm{I}\right.$ or $\left.\mathrm{CH}_{3}\right)$ reaction. An $a b$ initio study of the enthalpy of formation of the $\mathrm{CH}_{2} \mathrm{I}, \mathrm{CHI}_{2}$ and $\mathrm{CI}_{3}$ radicals. Phys. Chem. Chem. Phys., 2002, 4, 455-460.

(69) Chen E.C.M.; Albyn K.; Dussack I.; Wentworth W.E. Determination of bond dissociation energies from dissociative thermal electron attachment. J. Phys. Chem., 1989, 93, 6827-6832.

(70) McGivern S. W.; Derecskei-Kovacs A.; North S.W., Francisco J. S., Computationally efficient methodology to calculate $\mathrm{C}-\mathrm{H}$ and $\mathrm{C}-\mathrm{X}(\mathrm{X}=\mathrm{F}, \mathrm{Cl}$, and $\mathrm{Br})$ bond dissociation energies in haloalkanes. J. Phys. Chem. A, 2000, 104 (2), 436-442. 